\title{
Calabi quasi-morphisms for some non-monotone symplectic manifolds
}

\author{
YARON OSTROVER
}

\begin{abstract}
In this work we construct Calabi quasi-morphisms on the universal cover $\widetilde{\operatorname{Ham}}(M)$ of the group of Hamiltonian diffeomorphisms for some non-monotone symplectic manifolds. This complements a result by Entov and Polterovich which applies in the monotone case. Moreover, in contrast to their work, we show that these quasimorphisms descend to non-trivial homomorphisms on the fundamental group of $\operatorname{Ham}(M)$.
\end{abstract}

53D05, 53D12, 53D45; 20F69

\section{Introduction and results}

Let $(M, \omega)$ be a closed connected symplectic manifold of dimension $2 n$. Let $\operatorname{Ham}(M, \omega)$ denote the group of Hamiltonian diffeomorphisms of $(M, \omega)$ and let $\widetilde{\operatorname{Ham}}(M, \omega)$ be its universal cover. A celebrated result by Banyaga [3] states that for a closed symplectic manifold, $\operatorname{Ham}(M, \omega)$ and $\widetilde{\operatorname{Ham}}(M, \omega)$ are simple groups and therefore they do not admit any non-trivial homomorphism to $\mathbb{R}$. However, in some cases, these groups admit non-trivial homogeneous quasi-morphisms to $\mathbb{R}$. Recall that a (real-valued) quasi-morphism of a group $G$ is a map $r: G \rightarrow \mathbb{R}$ satisfying the homomorphism equation up to a bounded error, i.e. there exists a constant $C \geq 0$ such that

$$
\left|r\left(g_{1} g_{2}\right)-r\left(g_{1}\right)-r\left(g_{2}\right)\right| \leq C, \text { for every } g_{1}, g_{2} \in G \text {. }
$$

A quasi-morphism $r$ is called homogeneous if $r\left(g^{n}\right)=n r(g)$ for all $g \in G$ and $n \in \mathbb{Z}$. The existence of homogeneous quasi-morphisms on the group of Hamiltonian diffeomorphisms and/or its universal cover is known for some classes of closed symplectic manifolds (see e.g. Barge-Ghys [4], Entov [9], Gambaudo-Ghys [13] and Givental [14]). In a recent work [11], Entov and Polterovich showed - by using Floer and Quantum homology - that for the class of symplectic manifolds which are monotone and whose quantum homology algebra is semi-simple, $\widetilde{\operatorname{Ham}}(M, \omega)$ admits a homogeneous quasi-morphism to $\mathbb{R}$. In addition to constructing such a quasi-morphism, Entov and Polterovich showed that its value on any diffeomorphism 
supported in a Hamiltonianly displaceable open subset equals to the Calabi invariant of the diffeomorphism (see Section 2 below for precise definitions). A quasi-morphism with this property is called a Calabi quasi-morphism.

The notion "quasi-morphism" first appeared works of Brooks [7] and Gromov [15] on bounded cohomology of groups. Since then, quasi-morphisms have become an important tool in the study of groups. For example, the mere existence of a homogeneous quasi-morphism on a group $G$ which does not vanish on the commutator subgroup $G^{\prime}$ implies that the commutator subgroup has infinite diameter with respect to the commutator norm (see e.g. Bavard [5]). Two well known examples of quasimorphisms are the Maslov quasi-morphism on the universal cover of the group of linear symplectomorphisms of $\mathbb{R}^{2 n}$, and the rotation quasi-morphism defined on the universal cover of the group of orientation-preserving homeomorphisms of $S^{1}$. We refer the readers to Bavard [5] and Kotschick [18] and the references cited therein for further details on this subject. Recently, Biran, Entov and Polterovich [6], and Entov and Polterovich [10] established several other applications of the existence of a Calabi quasi-morphism regarding rigidity of intersections in symplectic manifolds. An example of this type is given in Theorem 1.5 and Corollary 1.6 below.

In view of the work by Entov and Polterovich [11], it is natural to ask which classes of symplectic manifolds admit a Calabi quasi-morphism. In a very recent work, Py [31] constructed a homogeneous Calabi quasi-morphism for closed oriented surfaces with genus greater than 1. In this note we concentrate on the case of non-monotone symplectic manifolds. We will provide some examples of non-monotone rational ruled surfaces admitting a Calabi quasi-morphism. More precisely, let

$$
X_{\lambda}=\left(S^{2} \times S^{2}, \omega_{\lambda}=\omega \oplus \lambda \omega\right), \quad 1 \leq \lambda \in \mathbb{R},
$$

where $\omega$ is the standard area form on the two-sphere $S^{2}$ with area 1 , and let

$$
Y_{\mu}=\left(\mathbb{C} P^{2} \# \overline{\mathbb{C} P^{2}}, \omega_{\mu}\right), \quad 0<\mu<1,
$$

be the symplectic blow-up of $\mathbb{C} P^{2}$ at one point (see e.g. McDuff [22], Polterovich [30]), where $\omega_{\mu}$ takes the value $\mu$ on the exceptional divisor, and 1 on the class of the line $\left[\mathbb{C} P^{1}\right]$. The manifold $Y_{\mu}$ is the region

$$
\left\{\left.\left(z_{1}, z_{2}\right) \in \mathbb{C}^{2}|\mu \leq| z_{1}\right|^{2}+\left|z_{2}\right|^{2} \leq 1\right\}
$$

with the bounding spheres collapsed along the Hopf flow. It is known that any symplectic form on these manifolds is, up to a scaling by a constant, diffeomorphic to one of the above symplectic forms (see Lalonde-McDuff [19]). 
In the monotone case where $\lambda=1$ and $\mu=\frac{1}{3}$, Entov and Polterovich [11] proved the existence of a homogeneous Calabi quasi-morphism on the universal covers of $\operatorname{Ham}\left(X_{\lambda}\right)$ and $\operatorname{Ham}\left(Y_{\mu}\right)$. Moreover, they shows that these quasi-morphisms are Lipschitz with respect to Hofer's metric. For the precise definition of the Lipschitz property of a quasi-morphism, see Section 2 below. Here we prove the following:

Theorem 1.1 Let $(M, \omega)$ be one of the following symplectic manifolds:

(i) $X_{\lambda}=\left(S^{2} \times S^{2}, \omega_{\lambda}\right)$, where $1<\lambda \in \mathbb{Q}$.

(ii) $Y_{\mu}=\left(\mathbb{C} P^{2} \# \overline{\mathbb{C} P^{2}}, \omega_{\mu}\right)$, where $\frac{1}{3} \neq \mu \in \mathbb{Q} \cap(0,1)$.

Then there exists a homogeneous Calabi quasi-morphism $\widetilde{r}: \widetilde{\operatorname{Ham}}(M, \omega) \rightarrow \mathbb{R}$, which is Lipschitz with respect to Hofer's metric.

It can be shown in the monotone case that any homogeneous quasi-morphism on the universal cover of $\operatorname{Ham}\left(X_{1}\right)$ descends to a quasi-morphism on $\operatorname{Ham}\left(X_{1}\right)$ itself [11]. This is due to the finiteness of the fundamental group $\pi_{1}\left(\operatorname{Ham}\left(X_{1}\right)\right)$, which was proved by Gromov in [16]. He also pointed out that the homotopy type of the group of symplectomorphisms of $S^{2} \times S^{2}$ changes when the spheres have different areas. McDuff [21], and Abreu and McDuff [1], showed that the fundamental groups, $\pi_{1}\left(\operatorname{Ham}\left(X_{\lambda}\right)\right)$ and $\pi_{1}\left(\operatorname{Ham}\left(Y_{\mu}\right)\right)$, contain elements of infinite order for every $0<\mu<1$ and for every $\lambda>1$. Thus, the above argument will no longer hold in these cases. Furthermore we claim:

Theorem 1.2 Let $M$ be one of the manifolds listed in Theorem 1.1. Then the restriction of the above mentioned Calabi quasi-morphism $\widetilde{r}: \widetilde{\operatorname{Ham}}(M, \omega) \rightarrow \mathbb{R}$ to the fundamental group $\pi_{1}(\operatorname{Ham}(M, \omega)) \subset \widetilde{\operatorname{Ham}}(M, \omega)$ gives rise to a non-trivial homomorphism.

This differs from the situation described in [11] where it was proven that for $M=\mathbb{C} P^{n}$ endowed with the Fubini-Study form, or for $M=S^{2} \times S^{2}$ equipped with the split symplectic form $\omega \oplus \omega$, the restriction of the Calabi quasi-morphism to the fundamental group $\pi_{1}(\operatorname{Ham}(M))$ vanishes identically.

For technical reasons, we shall assume in what follows that $M$ is a rational strongly semi-positive symplectic manifold. Recall that a symplectic manifold $M$ is rational if the set $\left\{\omega(A) \mid A \in \pi_{2}(M)\right\}$ is a discrete subset of $\mathbb{R}$ and strongly semi-positive if for every $A \in \pi_{2}(M)$ one has

$$
2-n \leq c_{1}(A)<0 \Longrightarrow \omega(A) \leq 0,
$$


where $c_{1} \in H^{2}(M, \mathbb{Z})$ denotes the first Chern class of $M$. The assumption that $M$ is strongly semi-positive is a standard technical assumption (see e.g. Piunikhin-SalamonSchwarz [29], Seidel [35]) which guarantees, roughly speaking, the good-behavior of some moduli spaces of pseudo-holomorphic curves. Note that every symplectic manifold of dimension 4 or less, in particular the manifolds listed in Theorem 1.1, is strongly semi-positive. The rationality assumption is also a technical assumption. It plays a role, for example, in Lemma 5.1 below, where for non-rational symplectic manifolds the action spectrum is a non-discrete subset of $\mathbb{R}$ and our method of proof fails.

In fact, the examples in Theorem 1.1 are special cases of a more general criterion for the existence of a Calabi quasi-morphism. In [11], such a criterion was given for closed monotone symplectic manifolds. This criterion is based on some algebraic properties of the quantum homology algebra of $(M, \omega)$. More precisely, recall that as a module the quantum homology of $M$ is defined as $Q H_{*}(M)=H_{*}(M) \otimes \Lambda$, where $\Lambda$ is the standard Novikov ring

$$
\Lambda=\left\{\sum_{A \in \Gamma} \lambda_{A} q^{A} \mid \lambda_{A} \in \mathbb{Q}, \#\left\{A \in \Gamma \mid \lambda_{A} \neq 0, \omega(A)>c\right\}<\infty, \forall c \in \mathbb{R}\right\} .
$$

Here $\Gamma=\pi_{2}(M) /\left(\operatorname{ker} c_{1} \cap \operatorname{ker} \omega\right)$, where $c_{1}$ is the first Chern class. A grading on $\Lambda$ is given by $\operatorname{deg}\left(q^{A}\right)=2 c_{1}(A)$. We shall denote by $\Lambda_{k}$ all the elements in $\Lambda$ with degree $k$. We refer the readers to McDuff-Salamon [23] and to Subsection 3.1 below for a more detailed exposition and for the precise definition of the quantum product on $Q H_{*}(M)$. In the monotone case, i.e. where there exists $\kappa>0$ such that $\omega=\kappa \cdot c_{1}$, the Novikov ring $\Lambda$ can be identified with the field of Laurent series $\sum \alpha_{j} x^{j}$, with coefficients in $\mathbb{Q}$, and all $\alpha_{j}$ vanish for $j$ greater than some large enough $j_{0}$. In this case we say that the quantum homology $Q H_{*}(M)$ is semi-simple if it splits with respect to multiplication into a direct sum of fields, all of which are finite dimension linear spaces over $\Lambda$. It was shown in [11] that for monotone symplectic manifolds with semi-simple quantum homology algebra there exists a Lipschitz homogenous Calabi quasi-morphism on the universal cover of the group of Hamiltonian diffeomorphisms.

In the non-monotone case the above definition of semi-simplicity will no longer hold since $\Lambda$ is no longer a field. However, it turns out that a similar criterion to the above still exists in this case. More precisely, we focus upon the sub-algebra $Q H_{2 n}(M) \subset$ $Q H_{*}(M)$ over the sub-ring $\Lambda_{0} \subset \Lambda$. This sub-algebra is the degree component of the identity in $Q H_{*}(M)$. Using the fact that in the non-monotone case the sub-ring $\Lambda_{0}$ can be identified with the field of Laurent series, we say as before that $Q H_{2 n}(M)$ is semi-simple over $\Lambda_{0}$ if it splits into a direct sum of fields with respect to multiplication. Denote by $N_{M}$ the minimal Chern number of $M$ defined as the positive generator of 
the image $c_{1}\left(\pi_{2}(M)\right) \subseteq \mathbb{Z}$ of the first Chern class $c_{1}$. The following criterion is a generalization of Theorem 1.5 from [11] to the rational strongly semi-positive case.

Theorem 1.3 Let $(M, \omega)$ be a closed connected rational strongly semi-positive symplectic manifold of dimension $2 n$. Suppose that the quantum homology subalgebra $Q H_{2 n}(M) \subset Q H_{*}(M)$ is a semi-simple algebra over the field $\Lambda_{0}$ and that $N_{M}$ divides $n$. Then there exists a Lipschitz homogeneous Calabi quasi-morphism $\widetilde{r}: \widetilde{\operatorname{Ham}}(M, \omega) \rightarrow \mathbb{R}$.

For the manifolds $X_{\lambda}$ and $Y_{\mu}$ listed in Theorem 1.1 the minimal Chern number $N_{M}$ is 2 and 1 respectively. Thus, one of our main tasks is to prove that for these manifolds the top-dimension quantum homology subalgebra $Q H_{4}(M)$ is semi-simple over the field $\Lambda_{0}$.

As a by-product of Theorem 1.1, we generalize a result regarding rigidity of intersections obtained by Entov and Polterovich in [10]. To describe the result, we recall first the following definitions. For a symplectic manifold $M$ denote by $\{\cdot, \cdot\}$ the standard Poisson brackets on $C^{\infty}(M)$. A linear subspace $\mathcal{A} \subset C^{\infty}(M)$ is said to be Poissoncommutative if $\{F, G\}=0$ for all $F, G \in \mathcal{A}$. We associate to a finite-dimensional Poisson-commutative subspace $\mathcal{A} \subset C^{\infty}(M)$ its moment map $\Phi_{\mathcal{A}}: M \rightarrow \mathcal{A}^{*}$, defined by $\left\langle\Phi_{\mathcal{A}}(x), F\right\rangle=F(x)$. A non-empty subset of the form $\Phi_{\mathcal{A}}^{-1}(p), p \in \mathcal{A}^{*}$, is called a fiber of $\mathcal{A}$. A fiber $X \subset M$ is said to be displaceable if there exists a Hamiltonian diffeomorphism $\varphi \in \operatorname{Ham}(M)$ such that $\varphi(X) \cap X=\varnothing$. The following definition was introduced in [10]:

Definition 1.4 A closed subset $X \subset M$ is called a stem, if there exists a finitedimensional Poisson-commutative subspace $\mathcal{A} \subset C^{\infty}(M)$, such that $X$ is a fiber of $\mathcal{A}$ and each fiber of $\mathcal{A}$, other than $X$, is displaceable.

In Theorem 2.4 of [10], Entov and Polterovich showed that for a certain class of symplectic manifolds, any two stems have a non-empty intersection. What they used, in fact, was only the existence of a Lipschitz homogeneous Calabi quasi-morphism for manifolds in this class. Using the exact same line of proof, the following theorem follows from Theorem 1.1 above.

Theorem 1.5 Let $M$ be one of the manifolds listed in Theorem 1.1. Then any two stems in $M$ intersect.

An example of a stem in the case where $M=X_{\lambda}$ is the product of two equators. More precisely, we identify $X_{\lambda}$ with $\mathbb{C} P^{1} \times \mathbb{C} P^{1}$ in the obvious way. Denote by $L \subset X_{\lambda}$ 
the Lagrangian torus defined by

$$
L=\left\{\left(\left[z_{0}: z_{1}\right],\left[w_{0}: w_{1}\right]\right) \in \mathbb{C} P^{1} \times \mathbb{C} P^{1}|| z_{0}|=| z_{1}|,| w_{0}|=| w_{1} \mid\right\}
$$

The proof that $L$ is a stem goes along the same line as Corollary 2.5 of [10]. Since the image of a stem under any symplectomorphism of $M$ is again a stem we get:

Corollary 1.6 Let $X_{\lambda}$ be one of the manifolds in the first class of manifolds listed in Theorem 1.1 above. Then for any symplectomorphism $\varphi$ of $X_{\lambda}$ we have $L \cap \varphi(L) \neq 0$.

Organization of the paper In Section 2 we recall some definitions and notations related to the Calabi quasi-morphism. In Section 3 we briefly review the definition of the quantum homology algebra $Q H_{*}(M)$. We then describe the quantum homologies of our main examples and state some of their properties. In Section 4 we recall the definition of Floer homology and some relevant notions. Section 5 is devoted to the proof of Theorem 1.1 and Theorem 1.3. In Section 6 we discuss the restriction of the Calabi quasi-morphisms on the fundamental group of $\operatorname{Ham}(M)$. In Section 7 we prove Theorem 1.2 and in the last section we prove the Poincare duality type lemma which is stated and applied in Section 5.

Acknowledgments I would like to thank my supervisor, Professor Leonid Polterovich, for his guidance, patience and invaluable advice during the preparation of this work. I would also like to thank Professor Michael Entov for many helpful suggestions. I am grateful to Professor Dusa McDuff for many useful remarks, and for inviting me to Stony Brook University and giving me the opportunity to present this work, as well as for her warm hospitality. A part of this work was done during my visit to the Max-Planck Institute in Leipzig. I thank the Max-Planck Institute for their invitation and especially I would like to thank Professor Matthias Schwarz for enlightening conversations and his warm hospitality. This research was partially supported by the Israel Science Foundation grant \# 11/03.

\section{Preliminaries on Calabi quasi-morphism}

In this section we recall the definition of a Calabi quasi-morphism introduced in [11]. We start with the definition of the classical Calabi invariant (see Banyaga [3] and Calabi [8]). Let $(M, \omega)$ be a closed connected symplectic manifold. Given a Hamiltonian function $H: S^{1} \times M \rightarrow \mathbb{R}$, set $H_{t}:=H(t, \cdot)$ and denote by $\varphi$ the time-1-map of the Hamiltonian flow $\left\{\varphi_{H}^{t}\right\}$. The group of Hamiltonian diffeomorphisms $\operatorname{Ham}(M, \omega)$ consists of all such time-1-maps. Let $\widetilde{\operatorname{Ham}}(M, \omega)$ be the universal cover of $\operatorname{Ham}(M, \omega)$. For a nonempty open subset $U$ of $M$, we denote by $\widetilde{\operatorname{Ham}}_{U}(M, \omega)$ the subgroup of $\widetilde{\operatorname{Ham}}(M, \omega)$, 
consisting of all elements that can be represented by a path $\left\{\varphi_{H}^{t}\right\}_{t \in[0,1]}$ starting at the identity and generated by a Hamiltonian function $H_{t}$ supported in $U$ for all $t$. For $\varphi \in \widetilde{\operatorname{Ham}}_{U}(M, \omega)$ we define $\mathrm{Cal}_{U}: \widetilde{\operatorname{Ham}}_{U}(M, \omega) \rightarrow \mathbb{R}$ by

$$
\varphi \mapsto \int_{0}^{1} d t \int_{M} H_{t} \omega^{n}
$$

This map is well defined, i.e. it is independent of the specific choice of the Hamiltonian function generating $\varphi$. Moreover, it is a group homomorphism called the Calabi homomorphism.

Recall that a non-empty subset $U$ of $M$ is called Hamiltonianly displaceable if there exists a Hamiltonian diffeomorphism $\varphi \in \operatorname{Ham}(M, \omega)$ such that $\varphi(U) \cap \operatorname{Closure}(U)=$ $\varnothing$. The following two definitions were introduced in [11].

Definition 2.1 A quasi-morphism on $\widetilde{\operatorname{Ham}}(M, \omega)$ coinciding with the Calabi homomorphism $\mathrm{Cal}_{U}: \widetilde{\operatorname{Ham}}_{U}(M, \omega) \rightarrow \mathbb{R}$ on any open and Hamiltonianly displaceable set $U$ is called a Calabi quasi-morphism.

Definition 2.2 A quasi-morphism $r: \widetilde{\operatorname{Ham}}(M, \omega) \rightarrow \mathbb{R}$ is said to be Lipschitz with respect to Hofer's metric if there exists a constant $K>0$ so that

$$
\left|r\left(\varphi_{H}\right)-r\left(\varphi_{F}\right)\right| \leq K \cdot\|H-F\|_{C^{0}}
$$

For the relation of $\|H-F\|_{C^{0}}$ to the Hofer distance between the corresponding Hamiltonian diffeomorphisms $\varphi_{H}$ and $\varphi_{H}$ see e.g. [11].

\section{The Quantum homology of our main examples}

\subsection{The quantum homology algebra}

In this section we briefly recall the definition of the quantum homology ring of $\left(M^{2 n}, \omega\right)$. We refer the readers to [23] for a detailed exposition on this subject. Let $M$ be a closed rational strongly semi-positive symplectic manifold of dimension $2 n$. By abuse of notation, we shall write $\omega(A)$ and $c_{1}(A)$ for the integrals of $\omega$ and $c_{1}$ over $A \in \pi_{2}(M)$. Let $\Gamma$ be the abelian group

$$
\Gamma=\pi_{2}(M) /\left(\operatorname{ker} c_{1} \cap \operatorname{ker} \omega\right) .
$$

We denote by $\Lambda$ the Novikov ring

$$
\Lambda=\left\{\sum_{A \in \Gamma} \lambda_{A} q^{A} \mid \lambda_{A} \in \mathbb{Q}, \#\left\{A \in \Gamma \mid \lambda_{A} \neq 0, \omega(A)>c\right\}<\infty, \forall c \in \mathbb{R}\right\} .
$$


This ring comes with a natural grading defined by $\operatorname{deg}\left(q^{A}\right)=2 c_{1}(A)$. We shall denote by $\Lambda_{k}$ all the elements of $\Lambda$ with degree $k$. Note that $\Lambda_{k}=\varnothing$ if $k$ is not an integer multiple of $2 N_{M}$, where $N_{M}$ is the minimal Chern number of $M$ defined by $c_{1}\left(\pi_{2}(M)\right)=N_{M} \mathbb{Z}$.

As a module, the quantum homology ring of $(M, \omega)$ is defined as

$$
Q H_{*}(M)=Q H_{*}(M, \Lambda)=H_{*}(M, \mathbb{Q}) \otimes \Lambda .
$$

A grading on $Q H_{*}(M)$ is given by $\operatorname{deg}\left(a \otimes q^{A}\right)=\operatorname{deg}(a)+2 c_{1}(A)$, where $\operatorname{deg}(a)$ is the standard degree of the class $a$ in the singular homology of $M$. Next, we define the quantum product on $Q H_{*}(M)$ (cf [23], [32]). For $a \in H_{i}(M)$ and $b \in H_{j}(M)$ we define $a * b \in Q H_{i+j-2 n}(M)$ as

$$
a * b=\sum_{A \in \Gamma}(a * b)_{A} \otimes q^{-A},
$$

where $(a * b)_{A} \in H_{i+j-2 n+2 c_{1}(A)}(M)$ is determined by the requirement that

$$
(a * b)_{A} \circ c=\Phi_{A}(a, b, c) \quad \text { for all } c \in H_{*}(M) .
$$

Here $\circ$ is the usual intersection product on $H_{*}(M)$, and $\Phi_{A}(a, b, c)$ denotes the Gromov-Witten invariant that counts the number of pseudo-holomorphic curves representing the class $A$ and intersecting with a generic representative of each of $a, b, c \in$ $H_{*}(M)$. The product $*$ is extended to $Q H_{*}(M)$ by linearity over the ring $\Lambda$. Note that the fundamental class $[M]$ is the unity with respect to the quantum multiplication.

It follows from the definitions that the zero-degree component of $a * b$ coincides with the classical cap-product $a \cap b$ in the singular homology. Moreover, there exists a natural pairing $\Delta: Q H_{k}(M) \times Q H_{2 n-k}(M) \rightarrow \Lambda_{0}$ defined by

$$
\Delta\left(\sum a_{A} \otimes q^{A}, \sum b_{B} \otimes q^{B}\right)=\sum_{c_{1}(A)=0}\left(\sum_{B}\left(a_{-B} \circ b_{B+A}\right)\right) q^{A} .
$$

The fact that the inner sums on the right hand side are always finite follows from the finiteness condition in (3.1.2). Moreover, the pairing $\Delta$ defines a Frobenius algebra structure, i.e. it is non-degenerate in the sense that $\Delta(\alpha, \beta)=0$ for all $\beta$ implies $\alpha=0$, and $\Delta(\alpha, \beta)=\Delta(\alpha * \beta,[M])$. Notice that $\Delta$ associates to each pair of quantum homology classes $\alpha, \beta \in Q H_{*}(M)$ the coefficient of the class $P=$ [point] in their quantum product. We also define a non-degenerate $\mathbb{Q}$-valued pairing $\Pi$ to be the zero order term of $\Delta$, i.e.

$$
\Pi\left(\sum a_{A} \otimes q^{A}, \sum b_{B} \otimes q^{B}\right)=\sum_{B}\left(a_{-B} \circ b_{B}\right) .
$$


Note that $\Pi(\alpha, \beta)=\Pi(\alpha * \beta,[M])$ for every pair of quantum homology classes $\alpha$ and $\beta$. Furthermore, the finiteness condition in the definition of the Novikov ring (3.1.2) leads to a natural valuation function val: $Q H_{*}(M) \rightarrow \mathbb{R}$ defined by

$$
\operatorname{val}\left(\sum_{A \in \Gamma} a_{A} \otimes q^{A}\right)=\max \left\{\omega(A) \mid a_{A} \neq 0\right\}, \text { and } \operatorname{val}(0)=-\infty .
$$

\subsection{The case of $S^{2} \times S^{2}$}

Let $X_{\lambda}=S^{2} \times S^{2}$ be equipped with the split symplectic form $\omega_{\lambda}=\omega \oplus \lambda \omega$, where $\lambda>1$. In this subsection we discuss several issues regarding the quantum homology of the manifold $X_{\lambda}$ and in particular we show that the quantum homology subalgebra $Q H_{4}\left(X_{\lambda}\right) \subset Q H_{*}\left(X_{\lambda}\right)$ is a field for every $\lambda>1$.

Denote the standard basis of $H_{*}\left(X_{\lambda}\right)$ by $P=$ [point], $A=\left[S^{2} \times\right.$ point], $B=$ [point $\times S^{2}$ ] and the fundamental class $M=\left[X_{\lambda}\right]$. The quantum homology of $X_{\lambda}$ is generated over the Novikov ring $\Lambda$ by these elements. Since $\lambda>1$, it follows that $\Gamma=\pi_{2}\left(X_{\lambda}\right)$, where the last is isomorphic to the free abelian group generated by $A$ and $B$. From the following Gromov-Witten invariants (see e.g. [11], [23]):

$$
\Phi_{A+B}(P, P, P)=1, \quad \Phi_{0}(A, B, M)=1, \quad \Phi_{A}(P, B, B)=1, \quad \Phi_{B}(P, A, A)=1,
$$

one finds the quantum identities:

$$
A * B=P, \quad A^{2}=M \otimes q^{-B}, \quad B^{2}=M \otimes q^{-A} .
$$

Next, instead of the standard basis $\{A, B\}$ of $\Gamma$, we consider the basis $\left\{e_{1}, e_{2}\right\}=$ $\{B-A, A\}$. Set $x=q^{e_{1}}$ and $y=q^{e_{2}}$. In this notation, the quantum product of the generators of $Q H_{*}\left(X_{\lambda}\right)$ becomes

$$
A * B=P, \quad A^{2}=M \otimes x^{-1} y^{-1}, \quad B^{2}=M \otimes y^{-1} .
$$

It follows from the definition of the Novikov ring (3.1.2) that

$$
\Lambda=\left\{\sum \lambda_{\alpha, \beta} \cdot x^{\alpha} y^{\beta} \mid \lambda_{\alpha, \beta} \in \mathbb{Q}\right\},
$$

where each sum satisfies the following finiteness condition:

$$
\#\left\{(\alpha, \beta) \mid \lambda_{\alpha, \beta} \neq 0, \alpha(\lambda-1)+\beta>c\right\}<\infty, \forall c \in \mathbb{R} .
$$

Taking into account the above mentioned grading of $\Lambda$ we get

$$
\begin{aligned}
\Lambda_{4 k} & =\left\{\sum \lambda_{\alpha, \beta} \cdot x^{\alpha} y^{\beta} \in \Lambda \mid 4 \beta=2 c_{1}\left(\alpha e_{1}+\beta e_{2}\right)=4 k\right\} \\
& =\left\{\sum \lambda_{\alpha} \cdot x^{\alpha} y^{k} \mid \#\left\{\alpha \mid \lambda_{\alpha} \neq 0, \alpha(\lambda-1)>d\right\}<\infty, \forall d \in \mathbb{R}\right\} .
\end{aligned}
$$


The finiteness condition above implies that $\lambda_{\alpha}$ vanishes for large enough $\alpha$ 's.

Lemma 3.1 For any $\lambda>1$, the subalgebra $Q H_{4}\left(X_{\lambda}\right) \subset Q H_{*}\left(X_{\lambda}\right)$ is a field.

Proof Let $0 \neq \gamma \in Q H_{4}\left(X_{\lambda}\right)$. Since $Q H_{4}\left(X_{\lambda}\right)=H_{4}\left(X_{\lambda}\right) \otimes \Lambda_{0}+H_{0}\left(X_{\lambda}\right) \otimes \Lambda_{4}$, it follows that

$$
\gamma=M \otimes \sum \lambda_{\alpha_{1}} x^{\alpha_{1}}+P \otimes y \sum \lambda_{\alpha_{2}} x^{\alpha_{2}}
$$

where $\lambda_{\alpha_{1}}$ and $\lambda_{\alpha_{2}}$ vanish for large enough $\alpha_{1}$ and $\alpha_{2}$ respectively. Next, let $\beta=P \otimes y$ be a formal variable. From the above multiplicative relations (3.2.2), we see that $\beta^{2}=M \otimes x^{-1}$. Hence, we can consider the following ring identification:

$$
Q H_{4}\left(X_{\lambda}\right) \simeq \mathcal{R}[\beta] / \mathcal{I}
$$

where $\mathcal{I}$ is the ideal generated by $\beta^{2}-x^{-1}$ and $\mathcal{R}=\mathbb{Q}[[x]$ is the ring of Laurent series $\sum \alpha_{j} x^{j}$, with coefficients in $\mathbb{Q}$, and all $\alpha_{j}$ vanish for $j$ greater than some large enough $j_{0}$. Note that for any Laurent series $\Phi(x) \in \mathcal{R}$, the maximal degree of $\Phi^{2}(x)$ is either zero or even. Therefore $\mathcal{R}$ does not contain a square root of $x^{-1}$ and hence $\mathcal{I}$ is a maximal ideal. Thus, we conclude that $Q H_{4}\left(X_{\lambda}\right)$ is a two-dimensional extension field of $\mathcal{R}$. This completes the proof of the lemma.

Remark 3.2 Note that the above statement no longer holds in the monotone case where $\lambda=1$, since $Q H_{4}\left(X_{1}\right)$ contains zero divisors (see e.g. [11], [23]).

\subsection{The case of $\mathbb{C} P^{2} \# \overline{\mathbb{C} P^{2}}$}

Here we study the quantum homology algebra of $Y_{\mu}=\left(\mathbb{C} P^{2} \# \overline{\mathbb{C} P^{2}}, \omega_{\mu}\right)$, which is the symplectic one-point blow-up of $\mathbb{C} P^{2}$ introduced in Section 1 . We will show that the quantum homology subalgebra $Q H_{4}\left(Y_{\mu}\right)$, which plays a central role in the proof of Theorem 1.1, is semi-simple. It is worth mentioning (see Remark 3.4 below) that the algebraic structure of $Q_{4}\left(Y_{\mu}\right)$ turns out to be dependent on $\mu$.

We denote by $E$ the exceptional divisor and by $L$ the class of the line $\left[\mathbb{C} P^{1}\right]$. Recall that for $0<\mu<1, \omega_{\mu}$ is a symplectic form on $Y_{\mu}$ with $\omega_{\mu}(E)=\mu$ and $\omega_{\mu}(L)=1$. Denote the class of a point by $P=$ [point] and set $F=L-E$. The elements $P, E, F$ and the fundamental class $M=\left[Y_{\mu}\right]$ form a basis of $H_{*}\left(Y_{\mu}\right)$.

The following description of the multiplicative relations for the generators of $Q H_{*}\left(Y_{\lambda}\right)$ can be found in [22].

$$
\begin{array}{ll}
P * P=(E+F) \otimes q^{-E-F}, & E * P=F \otimes q^{-F}, \\
P * F=M \otimes q^{-E-F}, & E * E=-P+E \otimes q^{-E}+M \otimes q^{-F}, \\
E * F=P-E \otimes q^{-E}, & F * F=E \otimes q^{-E} .
\end{array}
$$


Consider the rational non-monotone case where $\frac{1}{3} \neq \mu \in \mathbb{Q} \cap(0,1)$. Note that in this case $\Gamma \simeq \mathbb{Z} \otimes \mathbb{Z}$. As in the previous example of $S^{2} \times S^{2}$, we apply a unimodular change of coordinates and consider the following basis of $\Gamma$

$$
\Gamma \simeq \begin{cases}\operatorname{Span}_{\mathbb{Z}}\{F-2 E, E\}, & 0<\mu<\frac{1}{3} \\ \operatorname{Span}_{\mathbb{Z}}\{2 E-F, E\}, & \frac{1}{3}<\mu<1\end{cases}
$$

Denote $e_{1}=F-2 E, e_{2}=E$ when $0<\mu<\frac{1}{3}$ and $e_{1}=2 E-F, e_{2}=E$ when $\frac{1}{3}<\mu<1$. Set $x=q^{e_{1}}$ and $y=q^{e_{2}}$. From the definition of the Novikov ring (3.1.2) we have

$$
\Lambda=\left\{\sum \lambda_{\alpha, \beta} x^{\alpha} y^{\beta} \mid \lambda_{\alpha, \beta} \in \mathbb{Q}\right\},
$$

where each sum satisfies the following finiteness condition:

$$
\#\left\{(\alpha, \beta)\left|\lambda_{\alpha, \beta} \neq 0, \alpha\right| 3 \mu-1 \mid+\beta \mu>c\right\}<\infty, \forall c \in \mathbb{R} .
$$

The graded Novikov ring has the form

$$
\begin{aligned}
\Lambda_{2 i} & =\left\{\sum \lambda_{\alpha, \beta} \cdot x^{\alpha} y^{\beta} \in \Lambda \mid 2 \beta=2 c_{1}\left(\alpha e_{1}+\beta e_{2}\right)=2 i\right\} \\
& =\left\{\sum \lambda_{\alpha} \cdot x^{\alpha} y^{i} \mid \#\left\{\alpha\left|\lambda_{\alpha} \neq 0, \alpha\right| 3 \mu-1 \mid>d\right\}<\infty, \forall d \in \mathbb{R}\right\} .
\end{aligned}
$$

Next we present the quantum product of $Q H_{*}\left(Y_{\mu}\right)$ with respect to the above basis of $\Gamma$.

$$
\begin{array}{ll}
P * P=(E+F) \otimes x^{\kappa} y^{-3}, & E * P=F \otimes x^{\kappa} y^{-2}, \\
P * F=M \otimes x^{\kappa} y^{-3}, & E * E=-P+E \otimes y^{-1}+M \otimes x^{\kappa} y^{-2}, \\
E * F=P-E \otimes y^{-1}, & F * F=E \otimes y^{-1},
\end{array}
$$

where $\kappa=\operatorname{sgn}(3 \mu-1)$, i.e. $\kappa=1$ for $\frac{1}{3}<\mu<1$, and $\kappa=-1$ for $0<\mu<\frac{1}{3}$.

Lemma 3.3 The subalgebra $Q H_{4}\left(Y_{\mu}\right) \subset Q H_{*}\left(Y_{\mu}\right)$ is semi-simple.

Proof Since $Q H_{4}\left(Y_{\mu}\right)=H_{4}\left(Y_{\mu}\right) \otimes \Lambda_{0}+H_{2}\left(Y_{\mu}\right) \otimes \Lambda_{2}+H_{0}\left(Y_{\mu}\right) \otimes \Lambda_{4}$, it follows that for every $0 \neq \delta \in Q H_{4}\left(Y_{\mu}\right)$

$$
\begin{aligned}
\delta & =M \otimes \sum \lambda_{\alpha_{1}} x^{\alpha_{1}}+E \otimes y \sum \lambda_{\alpha_{2}} x^{\alpha_{2}} \\
& +F \otimes y \sum \lambda_{\alpha_{3}} x^{\alpha_{3}}+P \otimes y^{2} \sum \lambda_{\alpha_{4}} x^{\alpha_{4}},
\end{aligned}
$$

Algebraic $\mathcal{B}$ Geometric Topology, Volume 6 (2006) 
where $\lambda_{\alpha_{i}}$ vanish for large enough $\alpha_{i}$ for $i=1,2,3,4$. Next, put $\beta_{1}=E \otimes y$, $\beta_{2}=F \otimes y$ and $\beta_{3}=P \otimes y^{2}$. From the above multiplication table, we see that

$$
\left\{\begin{array}{l}
\beta_{1}^{2}=-\beta_{3}+\beta_{1}+x^{\kappa}, \\
\beta_{2}^{2}=\beta_{1}, \\
\beta_{3}^{2}=x^{\kappa}\left(\beta_{1}+\beta_{2}\right) \\
\beta_{1} \cdot \beta_{2}=\beta_{3}-\beta_{1}, \\
\beta_{2} \cdot \beta_{3}=x^{\kappa} \\
\beta_{1} \cdot \beta_{3}=x^{\kappa} \beta_{2} .
\end{array}\right.
$$

Thus, we have the following ring identification:

$$
Q H_{4}\left(Y_{\mu}\right) \simeq \mathcal{R}\left[\beta_{1}, \beta_{2}, \beta_{3}\right] / \mathcal{I},
$$

where $\mathcal{R}=\mathbb{Q}\left[[x]\right.$ is the ring of Laurent series $\sum \alpha_{j} x^{j}$ and $\mathcal{I}$ is the ideal generated by the above relations. It is easy to check that the sixth equation follows immediately from the second and the fifth equations and hence, it can be eliminated. Moreover, by isolating $\beta_{3}$ and $\beta_{1}$ from the first and the second equations respectively, we conclude that the above system is equivalent to the following one:

$$
\left\{\begin{array}{l}
\left(\beta_{2}^{2}-\beta_{2}^{4}+x^{\kappa}\right)^{2}=x^{\kappa}\left(\beta_{2}^{2}+\beta_{2}\right) \\
\beta_{2}^{3}=-\beta_{2}^{4}+x^{\kappa} \\
\beta_{2} \cdot\left(\beta_{2}^{2}-\beta_{2}^{4}+x^{\kappa}\right)=x^{\kappa}
\end{array}\right.
$$

Moreover, we claim that in fact

$$
Q H_{4}\left(Y_{\mu}\right) \simeq \mathcal{R}\left[\beta_{1}, \beta_{2}, \beta_{3}\right] / \mathcal{I} \simeq \mathcal{R}\left[\beta_{2}\right] / \mathcal{J}
$$

where $\mathcal{J}$ is the ideal generated by $\beta_{2}^{4}+\beta_{2}^{3}-x^{\kappa}$. Indeed, the first equation in (3.3.1) is obtained by multiplying the third equation by $\beta_{2}^{2}+\beta_{2}$ and assigning the second equation. The third equation is obtained from the second after multiplying it by $\beta_{2}-1$. Next, note that the polynomial $\beta_{2}^{4}+\beta_{2}^{3}-x^{\kappa}$ does not share a common root with its derivative since the roots of the derivative are 0 and $-3 / 4$. Thus, it has no multiple roots in $\mathcal{R}$ and hence the quantum homology subalgebra $Q H_{4}\left(Y_{\mu}\right)$ is semi-simple as required.

Remark 3.4 Strangely enough, it follows from the above lemma that the algebraic structure of the quantum homology subalgebra $Q H_{4}\left(Y_{\mu}\right)$ depends on $\mu$. More precisely, it can be shown that the polynomial $\beta_{2}^{4}+\beta_{2}^{3}-x^{\kappa}$ is irreducible over $\mathcal{R}$ for $\kappa=1$ while reducible for $\kappa=-1$. Thus, $Q H_{4}\left(Y_{\mu}\right)$ is a field when $\frac{1}{3}<\mu<1$, while for $0<\mu<\frac{1}{3}$, it is a direct sum of fields. We omit here the technical details because for our purpose, it is sufficient that $\mathrm{QH}_{4}\left(Y_{\mu}\right)$ is semi-simple. 


\section{Preliminaries on Floer homology}

In this section we give a brief review of Floer homology. In particular we present some definitions and notions which will be relevant for the proof of our main results. We refer the readers to [33] or [23] for a more detailed description.

Let $(M, \omega)$ be a closed, connected and strongly semi-positive symplectic manifold. Let $J=\left\{J_{t}\right\}_{0 \leq t \leq 1}$ be a periodic family of $\omega$-compatible almost complex structures. We denote by $\mathcal{L}$ the space of all smooth contractible loops $x: S^{1}=\mathbb{R} / \mathbb{Z} \rightarrow M$. Consider a covering $\widetilde{\mathcal{L}}$ of $\mathcal{L}$ whose elements are equivalence classes $[x, u]$ of pairs $(x, u)$, where $x \in \mathcal{L}, u$ is a disk spanning $x$ in $M$, and where

$$
\left(x_{1}, u_{1}\right) \sim\left(x_{2}, u_{2}\right) \text { if and only if } x_{1}=x_{2} \text { and } \omega\left(u_{1} \# u_{2}\right)=c_{1}\left(u_{1} \# u_{2}\right)=0 .
$$

The group of deck transformations of $\widetilde{\mathcal{L}}$ is naturally identified with the group $\Gamma$ (3.1.1), and we denote by

$$
[x, u] \mapsto[x, u \# A], \quad A \in \Gamma
$$

the action of $\Gamma$ on $\widetilde{\mathcal{L}}$. Moreover, we denote by $\mathcal{H}$ the set of all the zero-mean normalized Hamiltonian functions, i.e.

$$
\mathcal{H}=\left\{H \in C^{\infty}\left(S^{1} \times M\right) \mid \int_{M} H_{t} \omega^{n}=0, \text { for all } t \in[0,1]\right\} .
$$

For $H \in \mathcal{H}$, the symplectic action functional $\mathcal{A}_{H}: \widetilde{\mathcal{L}} \rightarrow \mathbb{R}$ is defined as

$$
\mathcal{A}_{H}([x, u]):=-\int_{u} \omega+\int_{S^{1}} H(x(t), t) d t .
$$

Note that

$$
\mathcal{A}_{H}([x, u \# A])=\mathcal{A}_{H}([x, u])-\omega(A) .
$$

Let $\mathcal{P}_{H}$ be the set of all contractible 1-periodic orbits of the Hamiltonian flow generated by $H$. Denote by $\widetilde{\mathcal{P}_{H}}$ the subset of pairs $[x, u] \in \widetilde{\mathcal{L}}$ where $x \in \mathcal{P}_{H}$. It is not difficult to verify that $\widetilde{\mathcal{P}_{H}}$ coincides with the set of critical points of $\mathcal{A}_{H}$. We define the action spectrum of $H$, denoted by $\operatorname{Spec}(H)$, as

$$
\operatorname{Spec}(H):=\left\{\mathcal{A}_{H}(x, u) \in \mathbb{R} \mid[x, u] \in \widetilde{\mathcal{P}_{H}}\right\} .
$$

Recall that the action spectrum is either a discrete or a countable dense subset of $\mathbb{R}$ [26].

We now turn to give the definition of the filtered Floer homology group. For a generic $H \in \mathcal{H}$ and $\alpha \in\{\mathbb{R} \backslash \operatorname{Spec}(H)\} \cup\{\infty\}$ define the vector space $C F_{k}^{\alpha}(H)$ to be

$$
C F_{k}^{\alpha}(H)=\left\{\sum_{[x, u] \in \widetilde{\mathcal{P}}_{(H)}} \beta_{[x, u]}[x, u] \mid \beta_{[x, u]} \in \mathbb{Q}, \mu([x, u])=k, \mathcal{A}_{H}([x, u])<\alpha\right\},
$$


where each sum satisfies the following finiteness condition:

$$
\#\left\{[x, u] \in \widetilde{\mathcal{P}_{H}} \mid \beta_{[x, u]} \neq 0 \text { and } \mathcal{A}_{H}([x, u])>\delta\right\}<\infty, \text { for every } \delta \in \mathbb{R} .
$$

Here $\mu([x, u])$ denotes the Conley-Zehnder index $\mu: \widetilde{\mathcal{P}_{H}} \rightarrow \mathbb{Z}$ (see e.g. [33]) which satisfies $\mu([x, u \# A])-\mu([x, u])=2 c_{1}(A)$. In particular, the Conley-Zehnder index of an element $x \in \mathcal{P}_{H}$ is well-defined modulo $2 N_{M}$, where $N_{M}$ is the minimal Chern number of $(M, \omega)$. The complex $C F_{k}^{\infty}(H)$ is a module over the Novikov ring $\Lambda$ (3.1.2), where the scalar multiplication of $\xi \in C F_{k}^{\infty}(H)$ with $\lambda \in \Lambda$ is given by

$$
\sum_{A} \sum_{[x, u]} a_{A} \cdot \alpha_{[x, u]}[x, u \# A] .
$$

For each given $[x, w]$ and $[y, v]$ in $\widetilde{\mathcal{P}_{H}}$, let $\mathcal{M}(H, J,[x, u],[y, v])$ be the moduli space of Floer connecting orbits from $[x, w]$ to $[y, v]$, i.e. the set of solutions $u: \mathbb{R} \times S^{1} \rightarrow M$ of the system

$$
\left\{\begin{array}{c}
\partial_{s} u+J_{t}(u)\left(\partial_{t} u-X_{H_{t}}(u)\right)=0, \\
\lim _{s \rightarrow-\infty} u(s, t)=x(t), \quad \lim _{s \rightarrow \infty} u(s, t)=y(t), \\
w \# u \# v \text { represent the trivial class in } \Gamma .
\end{array}\right\}
$$

It follows from the assumption of strongly semi-positivity and from Gromov's compactness theorem [16] that for a generic choice of $J$ the moduli spaces $\mathcal{M}([x, u],[y, v])$, for $\mu([x, u])-\mu([y, v])=1$, are compact.

The Floer boundary operator $\partial: C F_{k}^{\alpha}(H) \rightarrow C F_{k-1}^{\alpha}(H)$ is defined by

$$
\partial([x, w])=\sum n([x, w],[y, v])[y, v]
$$

where the sum runs over all the elements $[y, v] \in \widetilde{\mathcal{P}_{H}}$ such that $\mu[y, v]=k-1$ and $n([x, w],[y, v])$ denotes counting the (finitely many) un-parameterized Floer trajectories with a sign determined by a coherent orientation. As proved by Floer in [12], the boundary operator $\partial$ is well defined, satisfies $\partial^{2}=0$ and preserves the subspaces $C F_{*}^{\alpha}(H)$ (see [17]). Therefore, defining the quotient group by

$$
C F_{*}^{[a, b)}(H, J)=C F_{*}^{b}(H, J) / C F_{*}^{a}(H, J) \quad(-\infty<a \leq b \leq \infty),
$$

the boundary map induces a boundary operator $\partial: C F_{*}^{(a, b]}(H) \rightarrow C F_{*}^{(a, b]}(H)$, and we can define the Floer homology group by

$$
H F_{*}^{(a, b]}(J, H)=\left(C F_{*}^{(a, b]}(H), \partial\right) .
$$

We will use the convention $H F_{*}(H, J)=H F_{*}^{(-\infty, \infty]}(H, J)$ and $H F_{*}^{a}(H, J)=$ $H F_{*}^{(-\infty, a]}$. The graded homology $H F_{*}(H, J)$ is a module over the Novikov ring $\Lambda$, 
since the boundary operator is linear over $\Lambda$. Note that these homology groups have been defined for generic Hamiltonians only. However, one can extend the definition to all $H \in \mathcal{H}$ using a continuation procedure (see e.g [11]). A key observation is that the Floer homology groups are independent of the almost complex structure $J$ and the Hamiltonian $H$ used to define them. Moreover, if two Hamiltonian functions $H_{1}, H_{2} \in$ $\mathcal{H}$ generate the same element $\varphi \in \widetilde{\operatorname{Ham}}(M, \omega)$, then $\operatorname{Spec}\left(H_{1}\right)=\operatorname{Spec}\left(H_{2}\right)$ (see [25] and [34]) and the spaces $H F_{*}^{(a, b]}\left(J, H_{1}\right)$ and $H F_{*}^{(a, b]}\left(J, H_{2}\right)$ can be canonically identified. Therefore, we shall drop the notation $J$ and $H$ in $H F_{*}(H, J)$ and denote $H F_{*}(\varphi)=H F_{*}(J, H)$ where $\varphi \in \widetilde{\operatorname{Ham}}(M, \omega)$ is generated by $H$.

We denote by $\pi_{\alpha}: H F_{*}(\varphi) \rightarrow H F^{(\alpha, \infty]}(\varphi)$ the homomorphisms induced by the natural projection $C F_{\infty}(H) \rightarrow C F_{\infty}(H) / C F_{\alpha}(H)$ of Floer complexes and by $i_{\alpha}: H F_{\alpha}(\varphi) \rightarrow$ $H F_{*}(\varphi)$ the homomorphism induced by the inclusion map $i_{\alpha}: C F_{*}^{\alpha}(H) \rightarrow C F_{*}^{\infty}(H)$. Note that the homology long exact sequence yields Kernel $\pi_{\alpha}=$ Image $i_{\alpha}$. There exists a natural ring structure on the Floer homology groups named Pair-of-pants product (see e.g. [29])

$$
*_{\mathrm{pp}}: H F_{\alpha}(\varphi) \times H F_{\beta}(\psi) \rightarrow H F_{\alpha+\beta}(\varphi \psi) .
$$

In [29], Piunikhin, Salamon and Schwarz constructed a homomorphism between the Quantum homology groups $Q H_{*}(M)$ and the Floer homology groups $H F_{*}(M)$. Furthermore, they showed that the homomorphism $\Phi: Q H_{*}(M) \rightarrow H F_{*}(H)$ is an isomorphism which preserves the grading and intertwines the quantum product on $Q H_{*}(M)$ with the pair-of-pants product on $H F_{*}(H)$, i.e. $\Phi\left(i_{\alpha+\beta}\left(\xi *_{p p} \eta\right)\right)=$ $\Phi\left(i_{\alpha}(\xi)\right) * \Phi\left(i_{\beta}(\eta)\right)$, for every $\xi \in H F_{\alpha}(\varphi), \eta \in H F_{\beta}(\psi)$. In what follows, we will refer to the isomorphism $\Phi$ as the PSS isomorphism.

\section{The existence of a Calabi quasi-morphism}

Let $\left(M^{2 n}, \omega\right)$ be a closed connected rational strongly semi-positive symplectic manifold. Following the works of Viterbo [36], Schwarz [34], and Oh [27], we recall the definition of a spectral invariant $c$ which plays a central role in the proof of Theorem 1.3. We refer the readers to [27] and [23] for complete details of the construction and proofs of the general properties of this spectral invariant. A brief description of Floer homology and the PSS isomorphism was also given in the above Section 4.

We define the spectral invariant $c: Q H_{*}(M) \times \widetilde{\operatorname{Ham}}(M, \omega) \rightarrow \mathbb{R}$ as follows. For the elements $0 \neq a \in Q H_{*}(M)$ and $\varphi \in \widetilde{\operatorname{Ham}}(M, \omega)$, we set

$$
c(a, \varphi)=\inf \left\{\alpha \in \mathbb{R} \mid \Phi(a) \in \text { Image } i_{\alpha}\right\},
$$


where $\Phi: Q H_{*}(M) \rightarrow H F_{*}(\varphi)$ is the PSS isomorphism between the quantum homology and the Floer homology, and $i_{\alpha}: H F_{\alpha}(\varphi) \rightarrow H F_{*}(\varphi)$ is the natural inclusion in the filtered Floer homology. The non-trivial fact that $-\infty<c(a, \varphi)<\infty$ is proved in [27]. Moreover, $c(a, \varphi)$ has the following properties [27], [23]: For every $a, b \in Q H_{*}(M)$ and every $\varphi, \psi \in \widetilde{\operatorname{Ham}}(M)$

(P1) $c(a * b, \varphi \psi) \leq c(a, \varphi)+c(b, \psi)$,

(P2) $c(a, 11)=\operatorname{val}(a)$,

(P3) $c(a, \varphi)=\sup _{m} c\left(a^{[m]}, \varphi\right)$,

(P4) $c\left(a q^{A}, \varphi\right)=c(a, \varphi)+\omega(A)$, for every $q^{A} \in \Lambda$,

where $a^{[m]}$ is the grade- $m$-component of $a, \mathbb{1}$ is the identity in $\widetilde{\operatorname{Ham}}(M, \omega)$ and $\operatorname{val}(\cdot)$ is the valuation function (3.1.4) defined in Section 3.1.

The following lemma, which can be considered as a Poincaré duality type lemma, enables us to compare the spectral invariants of $\varphi$ and $\varphi^{-1}$. It is the analogue of Lemma 2.2 from [11] in the rational non-monotone case .

Lemma 5.1 For every $0 \neq \gamma \in Q H_{*}(M)$ and every $\varphi \in \widetilde{\operatorname{Ham}}(M, \omega)$

$$
c(\gamma, \varphi)=-\inf \left\{c\left(\delta, \varphi^{-1}\right) \mid \Pi(\delta, \gamma) \neq 0\right\},
$$

where $\Pi(\cdot, \cdot)$ is the $\mathbb{Q}$-valued pairing (3.1.3) defined in Section 3.1.

The proof of the lemma is given in Section 8 below. In order to prove Theorem 1.3 we will also need the following proposition. Assume that the subalgebra $Q H_{2 n}(M) \subset$ $Q H_{*}(M)$ is semi-simple over the field $\Lambda_{0}$ and let $Q H_{2 n}(M)=Q H_{2 n}^{1}(M) \oplus \cdots \oplus$ $Q_{2 n}^{k}(M)$ be a decomposition of $Q H_{2 n}(M)$ into a direct sum of fields. Then we have

Proposition 5.2 There exists a positive constant $C \in \mathbb{R}$ such that for every $0 \neq \gamma \in$ $Q H_{2 n}^{1}(M)$

$$
\operatorname{val}(\gamma)+\operatorname{val}\left(\gamma^{-1}\right) \leq C
$$

Postponing the proof of the above proposition we first present the proof of Theorem 1.3 and Theorem 1.1. In the proof of Theorem 1.3 we follow the strategy of the proof used by Entov and Polterovich in [11]. 
Proof of Theorem 1.3 Let $Q H_{2 n}(M)=Q H_{2 n}^{1}(M) \oplus \cdots \oplus Q_{2 n}^{k}(M)$ be a decomposition of $Q H_{2 n}(M)$ into a direct sum of fields. Consider the map $\widetilde{r}: \widetilde{\operatorname{Ham}}(M) \rightarrow \mathbb{R}$ defined by:

$$
\widetilde{r}(\varphi)=-\operatorname{vol}(M) \cdot \lim _{n \rightarrow \infty} \frac{c\left(e_{1}, \varphi^{n}\right)}{n},
$$

where $e_{1}$ is the unit element of $Q H_{2 n}^{1}(M)$. This is a standard homogenization of the map $c\left(e_{1}, \cdot\right): \widetilde{\operatorname{Ham}}(M) \rightarrow \mathbb{R}$. We claim that $\widetilde{r}$ is a Lipschitz homogenous Calabi quasi-morphism. The proof of the Calabi property and the Lipschitz property of $\widetilde{r}$ goes along the same lines as the proof of Propositions 3.3 and 3.5 in [11] with the notations suitably adapted. Thus, we will omit the details of the proof of these properties and concentrate on proving that $\widetilde{r}$ is a quasi-morphism. We will show that $c\left(e_{1}, \cdot\right)$ is a quasi-morphism, this immediately implies that its homogenization $\widetilde{r}$ is also a quasi-morphism.

Notice that the upper bound follows easily from the triangle inequality $(\mathrm{P} 1)$ :

$$
c\left(e_{1}, \varphi \psi\right)=c\left(e_{1} * e_{1}, \varphi \psi\right) \leq c\left(e_{1}, \varphi\right)+c\left(e_{1}, \psi\right) .
$$

Next, it follows from (P1) and Lemma 5.1 that

$$
c\left(e_{1}, \varphi\right) \leq c\left(e_{1}, \varphi \psi\right)+c\left(e_{1}, \psi^{-1}\right)=c\left(e_{1}, \varphi \psi\right)-\inf _{a: \Pi\left(a, e_{1}\right) \neq 0} c(a, \psi) .
$$

From the definition of the intersection pairing $\Pi$ (3.1.3) we have that

$$
\left\{a \mid \Pi\left(a, e_{1}\right) \neq 0\right\}=\left\{a \mid \Pi\left(a^{[0]}, e_{1}\right) \neq 0\right\}=\left\{a \mid \Pi\left(a^{[0]} * e_{1}, M\right) \neq 0\right\} .
$$

Combining this with the above property (P3) we may further estimate

$$
c\left(e_{1}, \varphi\right) \leq c\left(e_{1}, \varphi \psi\right)-\inf _{a: \Pi\left(a^{[0]} * e_{1}, M\right) \neq 0} c\left(a^{[0]}, \psi\right) .
$$

Our next step is to find a lower bound for the term $c\left(a^{[0]}, \psi\right)$ provided that $\Pi\left(a^{[0]} *\right.$ $\left.e_{1}, M\right) \neq 0$. For this, we shall first "shift" and then "project", roughly speaking, the element $a^{[0]} \in Q H_{0}(M)$ to the field $Q H_{2 n}^{1}(M)$. More precisely, since we assumed that the minimal Chern number $N_{M}$ divides $n$, there exists an element $q^{A}$ in the Novikov ring $\Lambda$ such that $a^{[0]} q^{A} \in Q H_{2 n}(M)$. Thus, it follows from properties (P1) and $(\mathrm{P} 4)$ that

$$
c\left(a^{[0]}, \psi\right)=c\left(a^{[0]} q^{A}, \psi\right)-\omega(A) \geq c\left(e_{1} * a^{[0]} q^{A}, \psi\right)-c\left(e_{1}, \mathbb{1}\right)-\omega(A) .
$$

Moreover, it follows from the assumption $\Pi\left(a^{[0]} * e_{1}, M\right) \neq 0$ and from the definition of the element $e_{1}$, that $e_{1} * a^{[0]} q^{A} \in Q H_{2 n}^{1}(M) \backslash\{0\}$. Hence, since $Q H_{2 n}^{1}(M)$ is a 
field, $e_{1} * a^{[0]} q^{A}$ is an invertible element inside it. Using the triangle inequality (P1) once again we get

$$
c\left(e_{1}, \psi\right) \leq c\left(e_{1} * a^{[0]} q^{A}, \psi\right)+c\left(\left(e_{1} * a^{[0]} q^{A}\right)^{-1}, 1\right) .
$$

Here $\left(e_{1} * a^{[0]} q^{A}\right)^{-1}$ is the inverse of $e_{1} * a^{[0]} q^{A}$ inside $Q H_{2 n}^{1}$. Next, by substituting this in the above inequality (5.2) and applying (P2) we can conclude

$$
c\left(a^{[0]}, \psi\right) \geq c\left(e_{1}, \psi\right)-\operatorname{val}\left(\left(e_{1} * a^{[0]} q^{A}\right)^{-1}\right)-\operatorname{val}\left(e_{1}\right)-\omega(A) .
$$

By assigning this lower bound of $c\left(a^{[0]}, \psi\right)$ into (5.1) we further conclude

$$
c\left(e_{1}, \varphi\right) \leq c\left(e_{1}, \varphi \psi\right)-c\left(e_{1}, \psi\right)+\sup _{a: \Pi\left(a^{[0]} * e_{1}, M\right) \neq 0} v a l\left(\left(e_{1} * a^{[0]} q^{A}\right)^{-1}\right)+C^{\prime},
$$

where $C^{\prime}$ is the value $\operatorname{val}\left(e_{1}\right)+\omega(A)$. The last step of the proof is to find a universal upper bound for $\operatorname{val}\left(\left(e_{1} * a^{[0]} q^{A}\right)^{-1}\right)$ provided that $\Pi\left(a^{[0]} * e_{1}, M\right) \neq 0$. Note that the condition $\Pi\left(a^{[0]} * e_{1}, M\right) \neq 0$ implies that $v a l\left(e_{1} * a^{[0]}\right) \geq 0$ and hence val $\left(e_{1} * a^{[0]} q^{A}\right) \geq-\omega(A)$. Therefore, it follows from Proposition 5.2 that $\operatorname{val}\left(\left(e_{1} *\right.\right.$ $\left.\left.a^{[0]} q^{A}\right)^{-1}\right)<C+\omega(A)$. We have shown that $c\left(e_{1}, \cdot\right)$ is a quasi-morphism, the proof of the theorem is thus complete.

Proof of Theorem 1.1 Let $M$ be one of the manifolds $\left(X_{\lambda}, \omega_{\lambda}\right)$ or $\left(Y_{\mu}, \omega_{\mu}\right)$ listed in the theorem. It follows from Lemma 3.1 and Lemma 3.3 that the subalgebra $Q H_{4}(M)$ is semi-simple. Moreover, the minimal Chern number of $\left(X_{\lambda}, \omega_{\lambda}\right)$ and $\left(Y_{\mu}, \omega_{\mu}\right)$ is 2 and 1 respectively. Thus, it follows from Theorem 1.3 that there exists a Lipschitz homogeneous Calabi quasi-morphism $r: \widetilde{\operatorname{Ham}}(M, \omega) \rightarrow \mathbb{R}$ as required.

Remark 5.3 As mentioned in Remark 3.4 above, in the case of $\left(Y_{\mu}, \omega_{\mu}\right)$ where $0<\mu<\frac{1}{3}$, the subalgebra $Q H_{4}\left(Y_{\mu}\right)$ splits into direct sum of two fields. Thus, using the units of these fields alternately, Theorem 1.3 implies the existence of two Calabi quasi-morphisms. We do not know whether they are equivalent or not.

We return now to the proof of Proposition 5.2. We will follow closely Lemma 3.2 in [11].

Proof of Proposition 5.2 From the definition of the graded Novikov ring it follows that $\Lambda_{0}$ can be identified with the field $\mathcal{R}=\mathbb{Q}\left[[x]\right.$ of Laurent series $\sum \alpha_{j} x^{j}$ with coefficients in $\mathbb{Q}$ and $\alpha_{j}=0$ for large enough $j$ 's. Moreover, it is not hard to check that $Q H_{k}(M)$ is a finite dimensional module over $\Lambda_{0}$. We denote by $\sigma: \mathcal{R} \rightarrow \mathbb{Z}$ the map which associates to a nonzero element $\sum \alpha_{j} x^{j} \in \mathcal{R}$ the maximal $j$, such 
that $\alpha_{j} \neq 0$. We set $\sigma(0)=-\infty$. For $\kappa \in \mathcal{R}$, put $|\kappa|_{1}=\exp \sigma(\kappa)$. Thus, $|\cdot|_{1}$ is a non-Archimedean absolute value on $\mathcal{R}$ and moreover, $\mathcal{R}$ is complete with respect to $|\cdot|_{1}$. For preliminaries on non-Archimedean geometry we refer the readers to [2]. Since the field $Q H_{2 n}^{1}(M)$ can be considered as a finite dimensional vector space over $\mathcal{R}$, the absolute value $|\cdot|_{1}$ can be extended to an absolute value $|\cdot|_{2}$ on $Q H_{2 n}^{1}(M)$ (see [2]). Note that $|\cdot|_{2}$ induces a multiplicative norm $\|\cdot\|_{2}$ on $Q H_{2 n}^{1}(M)$. On the other hand, we can consider a different norm on $Q H_{2 n}^{1}(M)$ defined by $\|\gamma\|_{3}=\exp \operatorname{val}(\gamma)$. Since all the norms on a finite dimensional vector space are equivalent, there is a constant $C_{1}>0$ such that

$$
\|\gamma\|_{3} \leq C_{1} \cdot\|\gamma\|_{2}, \text { for every } 0 \neq \gamma \in Q H_{2 n}^{1}(M) .
$$

Hence, for $0 \neq \gamma \in Q H_{2 n}^{1}(M)$, we have

$$
\|\gamma\|_{3} \cdot\left\|\gamma^{-1}\right\|_{3} \leq C_{1}^{2} \cdot\|\gamma\|_{2} \cdot\left\|\gamma^{-1}\right\|_{2}=C_{1}^{2} .
$$

Therefore, $\operatorname{val}(\gamma)+\operatorname{val}\left(\gamma^{-1}\right) \leq C$ where $C=2 \log C_{1}$. This completes the proof of the proposition.

\section{Restricting $\widetilde{r}$ to the fundamental group of $\operatorname{Ham}(M)$}

In this section we discuss the restriction of the above mentioned Calabi quasi-morphism $\widetilde{r}: \widetilde{\operatorname{Ham}}(M) \rightarrow \mathbb{R}$, where $M$ is one of the manifolds listed in Theorem 1.1, to the abelian subgroup $\pi_{1}(\operatorname{Ham}(\mathrm{M})) \subset \widetilde{\operatorname{Ham}}(M)$. For this purpose, we follow [11] and use the Seidel representation $\Psi: \pi_{1}(\operatorname{Ham}(M)) \rightarrow Q H_{e v}^{\times}(M, \mathbb{R})$ (see e.g. [35], [20]), where $Q H_{e v}^{\times}(M, \mathbb{R})$ denotes the group of units in the even part of the quantum homology algebra of $M$ with coefficients in a real Novikov ring. We start with the following preparation.

\subsection{Hamiltonian fibrations over the two sphere}

There is a one-to-one correspondence between homotopy classes of loops in $\operatorname{Ham}(M)$ and isomorphism classes of Hamiltonian fibrations over the two-sphere $S^{2}$ given by the following "clutching" construction (see e.g. [35], [20]). We assign to each loop $\varphi=\left\{\varphi_{t}\right\} \in \operatorname{Ham}(M)$ the bundle $(M, \omega) \rightarrow P_{\varphi} \rightarrow S^{2}$ obtained by gluing together the trivial fiber bundles $D^{ \pm} \times M$ along their boundary via $(t, x) \mapsto\left(t, \varphi_{t}(x)\right)$. Here we consider $S^{2}$ as $D^{+} \cup D^{-}$, where $D^{ \pm}$are closed discs with boundaries identified with $S^{1}$. Moreover, we orient the equator $D^{+} \cap D^{-}$as the boundary of $D^{+}$. Note that this correspondence can be reversed. 
As noted in [35], there are two canonical cohomology classes associated with such a fibration. One is the coupling class $u_{\varphi} \in H^{2}\left(P_{\varphi}, \mathbb{R}\right)$ which is uniquely defined by the following two conditions: the first is that it coincides with the class of the

symplectic form on each fiber, and the second is that its top power $u_{\varphi}^{n+1}$ vanishes. The other cohomology class is the first Chern class of the vertical tangent bundle $c_{\varphi}=c_{1}\left(T P_{\varphi}^{\text {vert }}\right) \in H^{2}\left(P_{\varphi}, \mathbb{R}\right)$. We define an equivalent relation on sections of the fibration $P_{\varphi} \rightarrow S^{2}$ in the following way: First, equip $S^{2}$ with a positive oriented complex structure $j$, and $P_{\varphi}$ with an almost complex structure $J$ such that the restriction of $J$ on each fiber is compatible with the symplectic form on it, and the projection $\pi: P_{\varphi} \rightarrow S^{2}$ is a $(J, j)$-holomorphic map. Next, two $(J, j)$-sections, $v_{1}$ and $\nu_{2}$, of $\pi: P_{\varphi} \rightarrow S^{2}$ are said to be $\Gamma$-equivalent if

$$
u_{\varphi}\left[v_{1}\left(S^{2}\right)\right]=u_{\varphi}\left[v_{2}\left(S^{2}\right)\right], \quad c_{\varphi}\left[v_{1}\left(S^{2}\right)\right]=c_{\varphi}\left[v_{2}\left(S^{2}\right)\right] .
$$

It has been shown in [35] that the set $\mathcal{S}_{\varphi}$ of all such equivalent classes is an affine space modeled on the group $\Gamma$ (3.1.1).

\subsection{The Seidel representation}

The following description of the Seidel representation, which is somehow different from Seidel's original work, can be found in [20]. For technical reasons, it will be more convenient to work in what follows with a slightly larger Novikov ring than in (3.1.2). More precisely, set $\mathcal{H}_{\mathbb{R}}:=H_{2}^{S}(M, \mathbb{R}) /\left(\operatorname{ker} c_{1} \cap \operatorname{ker} \omega\right)$, where $H_{2}^{S}(M, \mathbb{R})$ is the image of $\pi_{2}(M)$ in $H_{2}(M, \mathbb{R})$. We define the real Novikov ring as

$$
\Lambda_{\mathbb{R}}=\left\{\sum_{A \in \mathcal{H}_{\mathbb{R}}} \lambda_{A} q^{A} \mid \lambda_{A} \in \mathbb{Q}, \#\left\{A \in \Gamma \mid \lambda_{A} \neq 0, \omega(A)>c\right\}<\infty, \forall c \in \mathbb{R}\right\},
$$

and set $Q H_{*}(M):=Q H_{*}\left(M, \Lambda_{\mathbb{R}}\right)=H_{*}(M) \otimes \Lambda_{\mathbb{R}}$ to be the real quantum homology of $M$.

Next, let $\varphi$ be a loop of Hamiltonian diffeomorphisms and $v$ be an equivalence class of sections of $P_{\varphi}$. Set $d=2 c_{\varphi}(\nu)$. We define a $\Lambda_{\mathbb{R}}$-linear map $\Psi_{\varphi, \nu}: Q H_{*}(M) \rightarrow$ $Q H_{*+d}(M)$ as follows: for $a \in H_{*}(M, \mathbb{Z}), \Psi_{\varphi, v}$ is the class in $Q H_{*+d}(M)$ whose intersection with $b \in H_{*}(M, \mathbb{Z})$ is given by

$$
\Psi_{\varphi, v}(a) \cdot_{M} b=\sum_{B \in \mathcal{H}} n_{P_{\varphi}}(i(a), i(b) ; v+i(B)) q^{-B},
$$

where $i$ is the homomorphism $H_{*}(M) \rightarrow H_{*}\left(P_{\varphi}\right)$, the intersection $\cdot_{M}$ is the linear extension to $Q H_{*}(M)$ of the standard intersection pairing on $H_{*}(M, \mathbb{Q})$, and $n_{P_{\varphi}}(v, w ; \mu)$ is the Gromov-Witten invariant which counts isolated $J$-holomorphic stable curves in $P_{\varphi}$ of genus 0 and two marked points, such that each curve represents 
the equivalence class $\mu$ and whose marked points go through given generic representatives of the classes $v$ and $w$ in $H_{*}\left(P_{\varphi}, \mathbb{Z}\right)$. When the manifold $M$ is strongly semi-positive, these invariants are well defined. Moreover, it follows from Gromov's compactness theorem (see [16]) that for each given energy level $k$, there are only finitely many section-classes $\mu=v+i(B)$ with $\omega(B) \leq k$ that are represented by a $J$-holomorphic curve in $P_{\varphi}$. Thus, $\Psi_{\varphi, v}$ satisfies the finiteness condition for elements in $Q H_{*}(M)$.

For reasons of dimension, $n_{P_{\varphi}}(v, w ; \mu)=0$ unless $2 c_{\varphi}(\mu)+\operatorname{dim}(v)+\operatorname{dim}(w)=2 n$. Thus,

$$
\Psi_{\varphi, v}(a)=\sum a_{v, B} q^{-B}, a_{v, B} \in H_{*}(M),
$$

where $a_{v, B} \cdot M=n_{P_{\varphi}}(i(a), i(b) ; v+i(B))$, and

$$
\operatorname{dim}\left(a_{v, B}\right)=\operatorname{dim}(a)+2 c_{\varphi}(v+i(B))=\operatorname{dim}(a)+2 c_{\varphi}(v)+2 c_{1}(B) .
$$

Note also that $\Psi_{\varphi, v+A}=\Psi_{\varphi, v} \otimes q^{A}$. It has been shown by Seidel [35] (see also [20]) that $\Psi_{\varphi, v}$ is an isomorphism for all loops $\varphi$ and sections $v$.

Next, we use $\Psi_{\varphi, v}$ to define the Seidel representation

$$
\Psi: \pi_{1}(\operatorname{Ham}(M)) \rightarrow Q H_{*}\left(M, \Lambda_{\mathbb{R}}\right)^{\times} .
$$

In order to do so, we take a canonical section class $v_{\varphi}$ that (up to equivalence) satisfies the composition rule $v_{\varphi \psi}=v_{\psi} \# v_{\varphi}$, where $v_{\varphi \psi}$ denotes the obvious union of the sections in the fiber sum $P_{\psi \varphi}=P_{\psi} \# P_{\varphi}$. The section $v_{\varphi}$ is uniquely determined by the requirement that

$$
u_{\varphi}\left(v_{\varphi}\right)=0 \text { and } c_{\varphi}\left(v_{\varphi}\right)=0 .
$$

Moreover, it satisfies the above mentioned composition rule. Therefore, we get a group homomorphism

$$
\rho: \pi_{1}(\operatorname{Ham}(M)) \rightarrow \operatorname{Hom}_{\Lambda_{\mathbb{R}}}\left(Q H_{*}\left(M, \Lambda_{\mathbb{R}}\right)\right) .
$$

It has been shown in [35] that for all $\varphi \in \pi_{1}(\operatorname{Ham}(M))$ we have

$$
\rho(\varphi)(a)=\Psi_{\varphi}, v_{\varphi}([M]) *_{M} a .
$$

The Seidel representation is defined to be the natural homomorphism

$$
\Psi: \pi_{1}(\operatorname{Ham}(M)) \rightarrow Q H_{*}\left(M, \Lambda_{\mathbb{R}}\right)^{\times},
$$

given by $\varphi \mapsto \rho(\varphi)([M])$. 


\subsection{Relation with the spectral invariant}

Throughout, $\pi_{1}(\operatorname{Ham}(M))$ is considered as the group of all loops in $\operatorname{Ham}(M)$ based at the identity $\mathbb{1} \in \operatorname{Ham}(M)$. Let $\mathcal{L}$ be the space of all smooth contractible loops $x: S^{1}=\mathbb{R} / \mathbb{Z} \rightarrow M$ and $\widetilde{\mathcal{L}}$ its cover introduced in Section 4 . Let $\varphi$ be a loop of Hamiltonian diffeomorphisms. It is known (see e.g. [35]) that the orbits $\varphi_{t}(x)$ of $\varphi$ are contractible. We consider the map $T_{\varphi}: \mathcal{L} \rightarrow \mathcal{L}$ which takes the loop $x(t)$ to $\varphi_{t}(x(t))$. In [35], Seidel showed that this action can be lifted (not uniquely) to $\widetilde{\mathcal{L}}$. In fact it is not hard to check that there is a one-to-one correspondence between such lifts of $T_{\varphi}$ and equivalence classes of sections $v \in \mathcal{S}_{\varphi}$. We denote by $\widetilde{T}_{\varphi, v}$ the lift corresponding to $v \in \mathcal{S}_{\varphi}$. Next, let $\varphi \in \pi_{1}(\operatorname{Ham}(M))$ be a given loop generated by a normalized Hamiltonian $K \in \mathcal{H}$. The following formula, which can be found in [35] and [20], enables us to relate the Seidel representation with the spectral invariant $c$ used to define the Calabi quasi-morphism $\widetilde{r}$ :

$$
\left(\widetilde{T}_{\varphi, \nu}^{*}\right)^{-1} \mathcal{A}_{H}-\mathcal{A}_{K \sharp H}=-u_{\varphi}(\nu) \text {, for every } H \in \mathcal{H} .
$$

It has been shown in [35] that the isomorphism in the quantum homology level described in Section 6.2, which is obtained by multiplication with $\Psi_{\varphi}, v_{\varphi}([M])$ corresponds, under the identification between the Floer and the quantum homology, to the isomor-

phism $i: H F_{\alpha}(H) \rightarrow H F_{\alpha+u_{\varphi}(v)}(K \# H)$ induced by the action of $\left(\widetilde{T}_{\varphi, v}^{*}\right)$ on $\widetilde{\mathcal{L}}$. The following proposition can be found in [28] or [11].

Proposition 6.1 For every loop $[\varphi] \in \pi_{1}(\operatorname{Ham}(M)) \subset \widetilde{\operatorname{Ham}}(M)$ and every $a \in$ $Q H_{*}(M)$ we have

$$
c(a,[\varphi])=\operatorname{val}\left(a * \Psi([\varphi])^{-1}\right) .
$$

Proof Let $K \in \mathcal{H}$ be the normalized Hamiltonian function generating the loop $[\varphi]$, and let $H \in \mathcal{H}$ be the zero Hamiltonian generating the identity. The proposition immediately follows from (6.3.1) applied to $H$ and $K$.

\section{Proof of Theorem 1.2}

Recall that a homogeneous quasi-morphism on an abelian group is always a homomorphism (see e.g. [11]). Hence, in order to prove Theorem 1.2, we need to show that for the manifolds listed in the theorem, the restriction of the Calabi quasi-morphism $\widetilde{r}: \widetilde{\operatorname{Ham}}(M) \rightarrow \mathbb{R}$ on the fundamental group of $\operatorname{Ham}(M)$ is non-trivial. We will divide the proof into two parts.

Algebraic 83 Geometric Topology, Volume 6 (2006) 


\subsection{The case of $S^{2} \times S^{2}$.}

Let $X_{\lambda}=S^{2} \times S^{2}$ be equipped with the split symplectic form $\omega_{\lambda}=\omega \oplus \lambda \omega$, where $1<\lambda$. As mentioned in Section 1 , there is an element $[\varphi]$ of infinite order in the fundamental group of $\operatorname{Ham}\left(X_{\lambda}\right)$ (see [21]). This element can be represented by the following loop of diffeomorphisms

$$
\varphi_{t}(z, w)=\left(z, \Upsilon_{z, t}(w)\right),
$$

where $\Upsilon_{z, t}$ denotes the $2 \pi t$-rotation of the unit sphere $S^{2}$ around the axis through the points $z,-z$. Seidel showed in [35] (see also [24]), by direct calculation, that

$$
\Psi([\varphi])^{-1}=(A-B) \otimes q^{\alpha A+\beta B}\left(\sum_{j=0}^{\infty} q^{j(A-B)}\right),
$$

where $A$ and $B$ in $H_{*}\left(X_{\lambda}\right)$ are the classes of $\left[S^{2} \times\right.$ point] and [point $\left.\times S^{2}\right]$ respectively, and $\alpha, \beta \in \mathbb{R}$ were chosen such that $2 c_{1}(\alpha A+\beta B)=1$ and $\omega_{\lambda}(\alpha A+\beta B)=\frac{1}{2}+\frac{1}{6 \lambda}$.

Lemma 7.1 For every $n \in \mathbb{N}$ we have

$$
\operatorname{val}\left(\Psi([\varphi])^{-2 n}\right)=1+\frac{n}{3 \lambda} .
$$

Proof First note that $\operatorname{val}\left((A-B)^{2 n}\right)=\max \left\{\operatorname{val}\left(A^{k} B^{2 n-k}\right)\right\}$, where $0 \leq k \leq 2 n$. Next, set $\alpha_{k}=A^{k} B^{2 n-k}$. It follows from the quantum multiplication relations (3.2.1) that $\operatorname{val}\left(\alpha_{k+2}\right)=\operatorname{val}\left(\alpha_{k}\right)+(\lambda-1)$ for every $0 \leq k \leq 2 n-2$. Thus,

$$
\operatorname{val}\left((A-B)^{2 n}\right)=\max \left\{\operatorname{val}\left(A^{2 n}\right), \operatorname{val}\left(A^{2 n-1} B\right)\right\}=-n+1 .
$$

Set $\Delta=q^{\alpha A+\beta B}\left(\sum_{j=0}^{\infty} q^{j(A-B)}\right)$. It follow immediately that

$$
\operatorname{val}\left(\Delta^{2 n}\right)=\operatorname{val}\left(q^{2 n(\alpha A+\beta B)}\right)=2 n\left(\frac{1}{2}+\frac{1}{6 \lambda}\right)=n+\frac{n}{3 \lambda} .
$$

This completes the proof of the Lemma.

It follows from Lemma 3.1 that the subalgebra $Q H_{4}\left(X_{\lambda}\right)$ is a field. Thus, combining Proposition 6.1 and Lemma 7.1, we conclude that

$$
\widetilde{r}([\varphi])=-\operatorname{vol}\left(X_{\lambda}\right) \cdot \lim _{n \rightarrow \infty} \frac{\operatorname{val}\left(\Psi([\varphi])^{-2 n}\right)}{2 n}=-\frac{1+\lambda}{6 \lambda} \neq 0 .
$$

We have shown that the restriction of the quasi-morphism $\widetilde{r}: \widetilde{\operatorname{Ham}}\left(X_{\lambda}\right) \rightarrow \mathbb{R}$ on the fundametal group of $\operatorname{Ham}\left(X_{\lambda}\right)$ is non-trivial. This concludes the proof of Theorem 1.2 for the above case. 


\subsection{The case of $\mathbb{C} P^{2} \# \overline{\mathbb{C} P^{2}}$}

Let $Y_{\mu}=\mathbb{C} P^{2} \# \overline{\mathbb{C} P^{2}}$ be the symplectic one-point blow-up of $\mathbb{C} P^{2}$ introduced in Section 1 , equipped with the symplectic form $\omega_{\mu}$, where $\frac{1}{3} \neq \mu \in(0,1)$. We will use here the same notation as in Subsection 3.3. It has been shown by Abreu-McDuff in [1] that the fundamental group of $\operatorname{Ham}\left(Y_{\mu}\right)$ is isomorphic to $\mathbb{Z}$ with a generator given by the rotation

$$
\varphi:\left(z_{1}, z_{2}\right) \rightarrow\left(e^{-2 \pi i t} z_{1}, z_{2}\right), \quad 0 \leq t \leq 1 .
$$

The Seidel representation of $\varphi$ was computed in [22], [24] to be

$$
\Psi([\varphi])^{-1}=P \otimes q^{E / 2+3 F / 4-\delta(F-2 E)}, \quad \text { where } \delta=\frac{(1-\mu)^{2}}{12(1+\mu)(1-3 \mu)} .
$$

The following lemma can be immediately deduced from Lemma 5.1 and Remark 5.5 which both appear in [22].

Lemma 7.2 Let $\frac{1}{3} \neq \mu \in(0,1)$. Then

$$
\lim _{k \rightarrow \infty} \frac{\operatorname{val}\left(\Psi([\varphi])^{-k}\right)}{k}= \begin{cases}-\delta \omega(F-2 E), & \frac{1}{3}<\mu<1 \\ \frac{12-\delta}{12} \omega(F-2 E), & 0<\mu<\frac{1}{3}\end{cases}
$$

Proof Denote by $Q$ the element $P \otimes q^{E / 2+3 F / 4}$ and consider its powers $Q^{k}$ where $k \in \mathbb{N}$. It follows from the quantum multiplicative relations discussed in Subsection 3.2 that the only two possible cycles obtained by multiplication by $Q$ are

$$
P \otimes q^{E / 2+3 F / 4} \rightarrow E \otimes q^{F / 2} \rightarrow F \otimes q^{E / 2+F / 4} \rightarrow M \rightarrow P \otimes q^{E / 2+3 F / 4},
$$

and

$$
P \otimes q^{E / 2+3 F / 4} \rightarrow F \otimes q^{F / 2} \rightarrow M \otimes q^{F / 4-E / 2} \rightarrow P \otimes q^{F} .
$$

Thus, since the first cycle does not change the valuation, while the second cycle increases it by $\omega(F / 4-E / 2)$, we have that $\operatorname{val}\left(Q^{k}\right)$ is either bounded as $k \rightarrow \infty$ when $\omega(F / 4-E / 2)<0$ or linearly grows otherwise. Hence, we get that

$$
\operatorname{val}\left(\Psi([\varphi])^{-k}\right)= \begin{cases}C-\delta k \omega(F-2 E), & \frac{1}{3}<\mu<1 \\ C+\frac{k}{3} \omega(F / 4-E / 2)-\omega(\delta(F-2 E)), & 0<\mu<\frac{1}{3},\end{cases}
$$

where $C$ is some universal constant. This completes the proof. 
A straightforward calculation shows that the above expression (7.2.2) is strictly negative for every $0<\mu<1$. Thus, it follows from Proposition 6.1 and the fact that $\operatorname{val}(a * b) \leq$ $\operatorname{val}(a)+\operatorname{val}(b)$ that

$\widetilde{r}([\varphi])=-\operatorname{vol}\left(Y_{\mu}\right) \lim _{k \rightarrow \infty} \frac{\operatorname{val}\left(e_{1} * \Psi([\varphi])^{-k}\right)}{k} \geq-\operatorname{vol}\left(Y_{\mu}\right) \lim _{k \rightarrow \infty} \frac{\operatorname{val}\left(\Psi([\varphi])^{-k}\right)}{k}>0$

Hence, the restriction of the quasi-morphism $\widetilde{r}: \widetilde{\operatorname{Ham}}\left(Y_{\mu}\right) \rightarrow \mathbb{R}$ on the fundamental group of $\operatorname{Ham}\left(Y_{\mu}\right)$ is non-trivial. The proof of Theorem 1.2 is now complete.

\section{Proof of Lemma 5.1}

Let $(M, \omega)$ be a closed, rational and strongly semi-positive symplectic manifold of dimension $2 n$. Note that for rational symplectic manifolds the action spectrum is a discrete subset of $\mathbb{R}$, and thus there are only a finite number of critical values of the action functional $\mathcal{A}_{H}$ in any finite segment $[a, b] \subset \mathbb{R}$. Let $H(t, x) \in \mathcal{H}$ be a Hamiltonian function generating $\varphi \in \widetilde{\operatorname{Ham}}(M)$, and denote by $\widetilde{H}(t, x)=-H(-t, x)$ the Hamiltonian function generating the inverse symplectomorphism $\varphi^{-1}$. The set $\mathcal{P}_{H}$ of critical points of $\mathcal{A}_{H}$ is isomorphic to $\mathcal{P} \widetilde{H}$ via $\widetilde{x}(t)=x(-t)$, and $[x, u] \in \widetilde{\mathcal{P}_{H}}$ corresponds to $[\widetilde{x}, \widetilde{u}] \in \widetilde{\mathcal{P} \widetilde{H}}$ where

$\widetilde{u}(s, t)=u(-s,-t), \quad \mu([\widetilde{x}, \widetilde{u}])=2 n-\mu([x, u])$ and $\mathcal{A}_{H}([x, u])=-\mathcal{A}_{\bar{H}}([\widetilde{x}, \widetilde{u}])$.

We define a pairing $L: C F_{k}(H) \times C F_{2 n-k}(\widetilde{H}) \rightarrow \Lambda_{0}$ by

$$
L\left(\sum \alpha_{[x, u]} \cdot[x, u], \sum \beta_{[\widetilde{y}, \widetilde{v}]} \cdot[\widetilde{y}, \widetilde{v}]\right)=\sum_{A}\left(\sum_{[x, u]} \alpha_{[x, u]} \cdot \beta_{[x, u \sharp-A]}\right) q^{A},
$$

where the inner sum runs over all pairs $[x, u] \in \widetilde{\mathcal{P}_{H}}$ and the outer sum runs over all $A \in \Gamma$ with $c_{1}(A)=0$. The pairing $L$ is well defined. Indeed, consider first the inner sum, the finiteness condition in the definition of $C F_{*}(H)$ implies that it contains only finitely many elements. Secondly, it follows from the same reason that the power series on the right hand side of (8.1) satisfies the finiteness condition from the definition of the Novikov ring (3.1.2). It is not hard to check that the pairing $L$ is linear over $\Lambda_{0}$ and that it is non-degenerate in the standard sense. Thus, since the vector spaces $C F_{k}(H)$ and $C F_{2 n-k}(\widetilde{H})$ are finite dimensional over $\Lambda_{0}$, which is in our case a field, the pairing $L$ determines an isomorphism

$$
C F_{k}(H) \simeq \operatorname{Hom}_{\Lambda_{0}}\left(C F_{2 n-k}(\widetilde{H}), \Lambda_{0}\right) .
$$


From the universal coefficient theorem we obtain a Poincaré duality isomorphism

$$
H F_{k}(H) \simeq \operatorname{Hom}_{\Lambda_{0}}\left(H F_{2 n-k}(\widetilde{H}), \Lambda_{0}\right)
$$

In [29] it has been shown that the pairing determined by this isomorphism, which by abuse of notation we also denote by $L$, agrees with the intersection pairing $\Delta(\cdot, \cdot)$ on the quantum homology $Q H_{*}(M)$. More precisely, let $\Phi: Q H_{*}(M) \rightarrow H F_{*}(H)$ be the PSS isomorphism described in Section 4. Then, for every $a \in H F_{k}(H)$ and $b \in Q H_{2 n-k}(M)$ we have

$$
\Delta\left(\Phi^{-1}(a), b\right)=L(a, \Phi(b)) .
$$

Next, we consider the filtered Floer homology complexes $C F_{k}^{(-\infty, \alpha]}(H)$ and $C F_{2 n-k}^{(-\alpha, \infty]}(\widetilde{H})$. Note that these spaces are no longer vector spaces over $\Lambda_{0}$ since they are not closed with respect to the operation of multiplication by a scalar. We define a $\mathbb{Q}$-valued pairing $L^{\prime}: C F_{k}^{[-\infty, \alpha]}(H) \times C F_{2 n-k}^{(\alpha, \infty]}(\widetilde{H}) \rightarrow \mathbb{Q}$ by

$$
L^{\prime}\left(\sum \alpha_{[x, u]} \cdot[x, u], \sum \beta_{[\widetilde{y}, \widetilde{v}]} \cdot[\widetilde{y}, \widetilde{v}]\right)=\sum_{[x, u]}\left(\alpha_{[x, u]} \cdot \beta_{[x, u]}\right)
$$

This pairing is well defined since any element in $C F_{2 n-k}^{(-\alpha, \infty]}(\widetilde{H})$ is a finite sum. It is straightforward to check that the pairing $L^{\prime}$ is non-degenerate in the standard sense and that it coincides with the zero term of $L$. In other words, denote by $\tau: \Lambda_{0} \rightarrow \mathbb{Q}$ the map sending $\sum a_{A} q^{A}$ to $a_{0}$, then for any $a \in C F_{k}^{(-\infty, \alpha]}(H)$ and $b \in C F_{2 n-k}(\widetilde{H})$ we have

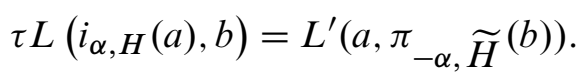

By abuse of notation, we also denote by $L^{\prime}$ the induced pairing in the homology level: $L^{\prime}: H F_{k}^{(-\infty, \alpha]}(H) \times H F_{2 n-k}^{(-\alpha, \infty]}(\widetilde{H}) \rightarrow \mathbb{Q}$. Next, consider the following diagram:

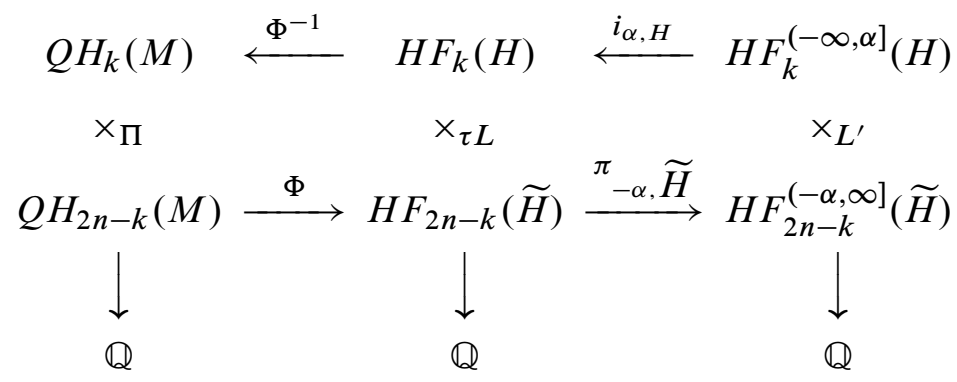

Algebraic 83 Geometric Topology, Volume 6 (2006) 
Combining equations (8.2) and (8.3) together we conclude that for every element $a \in H F_{k}^{(-\infty, \alpha]}(H)$ and $b \in Q H_{2 n-k}(M)$ we have

$$
\Pi\left(\Phi^{-1} \circ i_{\alpha, H}(a), b\right)=\tau L\left(i_{\alpha, H}(a), \Phi(b)\right)=L^{\prime}\left(a, \pi_{-\alpha, \widetilde{H}^{\circ}} \circ(b)\right)
$$

We are now in a position to prove Lemma 5.1.

Proof of Lemma 5.1 We divide the proof into two steps.

(1) Fix an arbitrary $\varepsilon>0$ and put $\alpha=\varepsilon-c(\gamma, \varphi)$. It follows from the definition of the spectral invariant $c$ that $\Phi(\gamma) \notin$ Image $i_{-\alpha, \varphi}$. Note that Image $i_{-\alpha, \varphi}=$ Kernel $\pi_{-\alpha, \varphi}$ and thus $\xi:=\pi_{-\alpha, \varphi} \circ \Phi(\gamma) \neq 0$. Since the pairing $L^{\prime}$ is nondegenerate there exists $\eta \in H F_{2 n-*}^{(-\infty, \alpha]}\left(\varphi^{-1}\right)$ such that $L^{\prime}(\eta, \xi) \neq 0$. From (8.4) we have that $\Pi\left(\delta_{0}, \gamma\right) \neq 0$, where $\delta_{0}=\Phi^{-1} \circ i_{\alpha, \varphi^{-1}}(\eta)$. It follows from the definition that $c\left(\delta_{0}, \varphi^{-1}\right) \leq \alpha$ and hence

$$
\inf _{\delta: \Pi(\delta, \gamma) \neq 0} c\left(\delta, \varphi^{-1}\right) \leq c\left(\delta_{0}, \varphi^{-1}\right) \leq \alpha=\varepsilon-c(\gamma, \varphi)
$$

This inequality holds for every $\varepsilon>0$, hence we conclude that

$$
\inf _{\delta: \Pi(\delta, \gamma) \neq 0} c\left(\delta, \varphi^{-1}\right) \leq-c(\gamma, \varphi) .
$$

(2) Fix an arbitrary $\varepsilon>0$ and put $\alpha=-\varepsilon-c(\gamma, \varphi)$. From the definition of $c(\cdot, \cdot)$ it follows that $\Phi(\gamma) \in$ Image $i_{-\alpha, \varphi}=$ Kernel $\pi_{-\alpha, \varphi}$. Hence, $\xi:=\pi_{-\alpha, \varphi} \circ \Phi(\gamma)=0$. Assume by contradiction that there exists $\delta$ satisfying $\Pi(\delta, \gamma) \neq 0$ such that $c\left(\delta, \varphi^{-1}\right)<\alpha$. We observe that $\Phi(\delta) \in$ Image $i_{\alpha, \varphi^{-1}}$. Let $\eta \in H F_{2 n-*}^{(-\infty, \alpha]}\left(\varphi^{-1}\right)$ be such that $\Phi(\delta)=i_{\alpha, \varphi^{-1}}(\eta)$. It follows from (8.4) that $\Pi(\delta, \gamma)=L^{\prime}(\eta, \xi)=0$. This contradicts the above assumption that $\Pi(\delta, \gamma) \neq 0$. Thus we must have $c\left(\delta, \varphi^{-1}\right) \geq \alpha$ for every $\delta$ satisfying $\Pi(\delta, \gamma) \neq 0$. Hence,

$$
\inf _{\delta: \Pi(\delta, \nu) \neq 0} c\left(\delta, \varphi^{-1}\right) \geq \alpha=-\varepsilon-c(\gamma, \varphi) .
$$

Again, since this inequality holds for every $\varepsilon>0$ we conclude that

$$
\inf _{\delta: \Pi(\delta, \gamma) \neq 0} c\left(\delta, \varphi^{-1}\right) \geq-c(\gamma, \varphi) .
$$

The proof is now complete. 


\section{References}

[1] M Abreu, D McDuff, Topology of symplectomorphism groups of rational ruled surfaces, J. Amer. Math. Soc. 13 (2000) 971-1009 MR1775741

[2] E Artin, Algebraic numbers and algebraic functions, Gordon and Breach Science Publishers, New York (1967) MR0237460

[3] A Banyaga, Sur la structure du groupe des difféomorphismes qui préservent une forme symplectique, Comment. Math. Helv. 53 (1978) 174-227 MR490874

[4] J Barge, É Ghys, Cocycles d'Euler et de Maslov, Math. Ann. 294 (1992) 235-265 MR1183404

[5] C Bavard, Longueur stable des commutateurs, Enseign. Math. (2) 37 (1991) 109-150 MR1115747

[6] P Biran, M Entov, L Polterovich, Calabi quasimorphisms for the symplectic ball, Commun. Contemp. Math. 6 (2004) 793-802 MR2100764

[7] R Brooks, Some remarks on bounded cohomology, from: "Riemann surfaces and related topics: Proceedings of the 1978 Stony Brook Conference (State Univ. New York, Stony Brook, N.Y., 1978)", Ann. of Math. Stud. 97, Princeton Univ. Press, Princeton, N.J. (1981) 53-63 MR624804

[8] E Calabi, On the group of automorphisms of a symplectic manifold, from: "Problems in analysis (Lectures at the Sympos. in honor of Salomon Bochner, Princeton Univ., Princeton, N.J., 1969)", Princeton Univ. Press, Princeton, N.J. (1970) 1-26 MR0350776

[9] M Entov, Commutator length of symplectomorphisms, Comment. Math. Helv. 79 (2004) 58-104 MR2031300

[10] M Entov, L Polterovich, Quasi-states and symplectic intersections arXiv: math.SG/0410338

[11] M Entov, L Polterovich, Calabi quasimorphism and quantum homology, Int. Math. Res. Not. (2003) 1635-1676 MR1979584

[12] A Floer, Symplectic fixed points and holomorphic spheres, Comm. Math. Phys. 120 (1989) 575-611 MR987770

[13] J-M Gambaudo, É Ghys, Commutators and diffeomorphisms of surfaces, Ergodic Theory Dynam. Systems 24 (2004) 1591-1617 MR2104597

[14] A B Givental, Nonlinear generalization of the Maslov index, from: "Theory of singularities and its applications", Adv. Soviet Math. 1, Amer. Math. Soc., Providence, RI (1990) 71-103 MR1089671

[15] M Gromov, Volume and bounded cohomology, Inst. Hautes Études Sci. Publ. Math. (1982) 5-99 (1983) MR686042 
[16] M Gromov, Pseudoholomorphic curves in symplectic manifolds, Invent. Math. 82 (1985) 307-347 MR809718

[17] H Hofer, D A Salamon, Floer homology and Novikov rings, from: "The Floer memorial volume”, Progr. Math. 133, Birkhäuser, Basel (1995) 483-524 MR1362838

[18] D Kotschick, What is .. a quasi-morphism?, Notices Amer. Math. Soc. 51 (2004) 208-209 MR2026941

[19] F Lalonde, D McDuff, The classification of ruled symplectic 4-manifolds, Math. Res. Lett. 3 (1996) 769-778 MR1426534

[20] F Lalonde, D McDuff, L Polterovich, Topological rigidity of Hamiltonian loops and quantum homology, Invent. Math. 135 (1999) 369-385 MR1666763

[21] D McDuff, Examples of symplectic structures, Invent. Math. 89 (1987) 13-36 MR892186

[22] D McDuff, Geometric variants of the Hofer norm, J. Symplectic Geom. 1 (2002) 197-252 MR1959582

[23] D McDuff, D Salamon, J-holomorphic curves and symplectic topology, American Mathematical Society Colloquium Publications 52, American Mathematical Society, Providence, RI (2004) MR2045629

[24] D McDuff, S Tolman, Topological properties of Hamiltonian circle actions arXiv: math.SG/0404338

[25] Y-G Oh, Mini-max theory, spectral invariants and geometry of the Hamiltonian diffeomorphism group arXiv:math.SG/0206092

[26] Y-G Oh, Chain level Floer theory and Hofer's geometry of the Hamiltonian diffeomorphism group, Asian J. Math. 6 (2002) 579-624 MR1958084

[27] Y-G Oh, Construction of spectral invariants of Hamiltonian paths on closed symplectic manifolds, from: "The breadth of symplectic and Poisson geometry", Progr. Math. 232, Birkhäuser, Boston (2005) 525-570 MR2103018

[28] Y-G Oh, Normalization of the Hamiltonian and the action spectrum, J. Korean Math. Soc. 42 (2005) 65-83 MR2106281

[29] S Piunikhin, D Salamon, M Schwarz, Symplectic Floer-Donaldson theory and quantum cohomology, from: "Contact and symplectic geometry (Cambridge, 1994)", Publ. Newton Inst. 8, Cambridge Univ. Press, Cambridge (1996) 171-200 MR1432464

[30] L Polterovich, Hamiltonian loops and Arnold's principle, from: "Topics in singularity theory”, Amer. Math. Soc. Transl. Ser. 2 180, Amer. Math. Soc., Providence, RI (1997) 181-187 MR1767123

[31] P Py, Quasi-morphismes et invariant de Calabi arXiv:math.SG/0506096

[32] Y Ruan, G Tian, A mathematical theory of quantum cohomology, J. Differential Geom. 42 (1995) 259-367 MR1366548 
[33] D Salamon, Lectures on Floer homology, from: "Symplectic geometry and topology (Park City, UT, 1997)", IAS/Park City Math. Ser. 7, Amer. Math. Soc., Providence, RI (1999) 143-229 MR1702944

[34] M Schwarz, On the action spectrum for closed symplectically aspherical manifolds, Pacific J. Math. 193 (2000) 419-461 MR1755825

[35] P Seidel, $\pi_{1}$ of symplectic automorphism groups and invertibles in quantum homology rings, Geom. Funct. Anal. 7 (1997) 1046-1095 MR1487754

[36] C Viterbo, Symplectic topology as the geometry of generating functions, Math. Ann. 292 (1992) 685-710 MR1157321

School of Mathematical Sciences, Tel Aviv University 69978 Tel Aviv, Israel

yaronost@post.tau.ac.il

Received: 9 September 2005 